\title{
The Universal Askey-Wilson Algebra and DAHA of Type $\left(C_{1}^{\vee}, C_{1}\right)$
}

\author{
Paul TERWILLIGER
}

Department of Mathematics, University of Wisconsin, Madison, WI 53706-1388, USA

E-mail: terwilli@math.wisc.edu

Received December 22, 2012, in final form July 07, 2013; Published online July 15, 2013

http://dx.doi.org/10.3842/SIGMA.2013.047

Abstract. Let $\mathbb{F}$ denote a field, and fix a nonzero $q \in \mathbb{F}$ such that $q^{4} \neq 1$. The universal Askey-Wilson algebra $\Delta_{q}$ is the associative $\mathbb{F}$-algebra defined by generators and relations in the following way. The generators are $A, B, C$. The relations assert that each of

$$
A+\frac{q B C-q^{-1} C B}{q^{2}-q^{-2}}, \quad B+\frac{q C A-q^{-1} A C}{q^{2}-q^{-2}}, \quad C+\frac{q A B-q^{-1} B A}{q^{2}-q^{-2}}
$$

is central in $\Delta_{q}$. The universal DAHA $\hat{H}_{q}$ of type $\left(C_{1}^{\vee}, C_{1}\right)$ is the associative $\mathbb{F}$-algebra defined by generators $\left\{t_{i}^{ \pm 1}\right\}_{i=0}^{3}$ and relations (i) $t_{i} t_{i}^{-1}=t_{i}^{-1} t_{i}=1$; (ii) $t_{i}+t_{i}^{-1}$ is central; (iii) $t_{0} t_{1} t_{2} t_{3}=q^{-1}$. We display an injection of $\mathbb{F}$-algebras $\psi: \Delta_{q} \rightarrow \hat{H}_{q}$ that sends

$$
A \mapsto t_{1} t_{0}+\left(t_{1} t_{0}\right)^{-1}, \quad B \mapsto t_{3} t_{0}+\left(t_{3} t_{0}\right)^{-1}, \quad C \mapsto t_{2} t_{0}+\left(t_{2} t_{0}\right)^{-1} .
$$

For the map $\psi$ we compute the image of the three central elements mentioned above. The algebra $\Delta_{q}$ has another central element of interest, called the Casimir element $\Omega$. We compute the image of $\Omega$ under $\psi$. We describe how the Artin braid group $B_{3}$ acts on $\Delta_{q}$ and $\hat{H}_{q}$ as a group of automorphisms. We show that $\psi$ commutes with these $B_{3}$ actions. Some related results are obtained.

Key words: Askey-Wilson polynomials; Askey-Wilson relations; rank one DAHA

2010 Mathematics Subject Classification: 33D80; 33D45

\section{Introduction}

The Askey-Wilson polynomials were introduced in [2] and soon became a major topic in special functions [6,9]. This topic became linked to representation theory through the work of A. Zhedanov [19]. In that article Zhedanov introduced the Askey-Wilson algebra AW(3), and showed that its "ladder" representations give the Askey-Wilson polynomials. The algebra $\mathrm{AW}(3)$ is noncommutative and infinite-dimensional. It is defined by generators and relations. The relations involve a scalar parameter $q$ and a handful of extra scalar parameters. The number of extra parameters ranges from 3 to 7 depending on which normalization is used [12, equation (6.1)], [17, Theorem 1.5], [18, Section 4.3], [19, equations (1.1a)-(1.1c)]. In [15] we introduced a central extension of $\mathrm{AW}(3)$, denoted $\Delta_{q}$ and called the universal Askey-Wilson algebra. Up to normalization $\Delta_{q}$ is obtained from $\mathrm{AW}(3)$ by interpreting the extra parameters as central elements in the algebra. By construction $\Delta_{q}$ has no scalar parameters besides $q$, and there is a surjective algebra homomorphism $\Delta_{q} \rightarrow \mathrm{AW}(3)$. One advantage of $\Delta_{q}$ over $\mathrm{AW}(3)$ is that $\Delta_{q}$ has a larger automorphism group. Our definition of $\Delta_{q}$ was inspired by [7, Section 3], which in turn was motivated by [5].

In [15] we began a comprehensive investigation of $\Delta_{q}$. In that paper we focused on its ringtheoretic aspects, and in a followup paper [16] we related $\Delta_{q}$ to the quantum algebra $U_{q}\left(\mathfrak{s l}_{2}\right)$. 
In the present paper we relate $\Delta_{q}$ to the universal DAHA of type $\left(C_{1}^{\vee}, C_{1}\right)$ [7]. This topic can be viewed as a universal analog of a topic considered by Koornwinder [10, 11], concerning how $\mathrm{AW}(3)$ is related to the DAHA of type $\left(C_{1}^{\vee}, C_{1}\right)$. We view [10,11] as groundbreaking and the main inspiration for the present paper. In Section 16 we describe in detail how our results relate to those of Koornwinder [10, 11].

We will state our main results after we summarize the contents of $[15,16]$.

Our conventions for the paper are as follows. An algebra is meant to be associative and have a 1. A subalgebra has the same 1 as the parent algebra. Fix a field $\mathbb{F}$. All unadorned tensor products are meant to be over $\mathbb{F}$. We fix a nonzero $q \in \mathbb{F}$ such that $q^{4} \neq 1$. Recall the natural numbers $\mathbb{N}=\{0,1,2, \ldots\}$ and integers $\mathbb{Z}=\{0, \pm 1, \pm 2, \ldots\}$.

The universal Askey-Wilson algebra $\Delta_{q}$ is the $\mathbb{F}$-algebra defined by generators and relations in the following way. The generators are $A, B, C$. The relations assert that each of

$$
A+\frac{q B C-q^{-1} C B}{q^{2}-q^{-2}}, \quad B+\frac{q C A-q^{-1} A C}{q^{2}-q^{-2}}, \quad C+\frac{q A B-q^{-1} B A}{q^{2}-q^{-2}}
$$

is central in $\Delta_{q}$. For the central elements (1.1) multiply each by $q+q^{-1}$ to get $\alpha, \beta, \gamma$. In [15] we obtained the following results about $\Delta_{q}$. We gave an alternate presentation for $\Delta_{q}$ by generators and relations; the generators are $A, B, \gamma$. We gave a faithful action of the modular group $\operatorname{PSL}_{2}(\mathbb{Z})$ on $\Delta_{q}$ as a group of automorphisms; one generator sends $(A, B, C) \mapsto(B, C, A)$ and another generator sends $(A, B, \gamma) \mapsto(B, A, \gamma)$. We showed that $\left\{A^{i} B^{j} C^{k} \alpha^{r} \beta^{s} \gamma^{t} \mid i, j, k, r, s, t \in \mathbb{N}\right\}$ is a basis for the $\mathbb{F}$-vector space $\Delta_{q}$. We showed that the center $Z\left(\Delta_{q}\right)$ contains a Casimir element

$$
\Omega=q^{-1} A C B+q^{-2} A^{2}+q^{-2} B^{2}+q^{2} C^{2}-q^{-1} A \alpha-q^{-1} B \beta-q C \gamma .
$$

Under the assumption that $q$ is not a root of unity, we showed that $Z\left(\Delta_{q}\right)$ is generated by $\Omega$, $\alpha, \beta, \gamma$ and that $Z\left(\Delta_{q}\right)$ is isomorphic to a polynomial algebra in four variables.

In [16] we relate $\Delta_{q}$ to the quantum algebra $U_{q}\left(\mathfrak{s l}_{2}\right)$. To describe this relationship we use the equitable presentation for $U_{q}\left(\mathfrak{s l}_{2}\right)$ which was introduced in [8]. This equitable presentation has generators $x, y^{ \pm 1}, z$ and relations $y y^{-1}=y^{-1} y=1$,

$$
\frac{q x y-q^{-1} y x}{q-q^{-1}}=1, \quad \frac{q y z-q^{-1} z y}{q-q^{-1}}=1, \quad \frac{q z x-q^{-1} x z}{q-q^{-1}}=1 .
$$

Let $a, b, c$ denote mutually commuting indeterminates. Let $\mathbb{F}\left[a^{ \pm 1}, b^{ \pm 1}, c^{ \pm 1}\right]$ denote the $\mathbb{F}$-algebra of Laurent polynomials in $a, b, c$ that have all coefficients in $\mathbb{F}$. In [16, Theorems 2.16, 2.18] we displayed an injection of $\mathbb{F}$-algebras $\sharp: \Delta_{q} \rightarrow U_{q}\left(\mathfrak{s l}_{2}\right) \otimes \mathbb{F}\left[a^{ \pm 1}, b^{ \pm 1}, c^{ \pm 1}\right]$ that sends

$$
\begin{aligned}
& A \mapsto x \otimes a+y \otimes a^{-1}+\frac{x y-y x}{q-q^{-1}} \otimes b c^{-1}, \quad B \mapsto y \otimes b+z \otimes b^{-1}+\frac{y z-z y}{q-q^{-1}} \otimes c a^{-1}, \\
& C \mapsto z \otimes c+x \otimes c^{-1}+\frac{z x-x z}{q-q^{-1}} \otimes a b^{-1} .
\end{aligned}
$$

The map $\downarrow$ sends

$$
\begin{aligned}
& \alpha \mapsto \Lambda \otimes\left(a+a^{-1}\right)+1 \otimes\left(b+b^{-1}\right)\left(c+c^{-1}\right), \\
& \beta \mapsto \Lambda \otimes\left(b+b^{-1}\right)+1 \otimes\left(c+c^{-1}\right)\left(a+a^{-1}\right), \\
& \gamma \mapsto \Lambda \otimes\left(c+c^{-1}\right)+1 \otimes\left(a+a^{-1}\right)\left(b+b^{-1}\right),
\end{aligned}
$$

where $\Lambda=q x+q^{-1} y+q z-q x y z$ is the normalized Casimir element of $U_{q}\left(\mathfrak{s l}_{2}\right)$ [16, Section 2]. In [16, Theorem 2.17] we showed that $\downarrow$ sends $\Omega$ to

$$
\begin{aligned}
& 1 \otimes\left(q+q^{-1}\right)^{2}-\Lambda^{2} \otimes 1-1 \otimes\left(a+a^{-1}\right)^{2}-1 \otimes\left(b+b^{-1}\right)^{2}-1 \otimes\left(c+c^{-1}\right)^{2} \\
& -\Lambda \otimes\left(a+a^{-1}\right)\left(b+b^{-1}\right)\left(c+c^{-1}\right) .
\end{aligned}
$$


We now summarize the present paper. We first show that the following is a basis for the $\mathbb{F}$-vector space $\Delta_{q}$ :

$$
A^{i} C^{j} B^{k} \Omega^{\ell} \alpha^{r} \beta^{s} \gamma^{t}, \quad j \in\{0,1\}, \quad i, k, \ell, r, s, t \in \mathbb{N} .
$$

This basis plays a role in our main topic, which is about how $\Delta_{q}$ is related to the universal DAHA $\hat{H}_{q}$ of type $\left(C_{1}^{\vee}, C_{1}\right)$. The algebra $\hat{H}_{q}$ is a variation on an algebra $\hat{H}$ introduced in [7]. By definition $\hat{H}_{q}$ is the $\mathbb{F}$-algebra with generators $\left\{t_{i}^{ \pm 1}\right\}_{i=0}^{3}$ and relations (i) $t_{i} t_{i}^{-1}=t_{i}^{-1} t_{i}=1$; (ii) $t_{i}+t_{i}^{-1}$ is central; (iii) $t_{0} t_{1} t_{2} t_{3}=q^{-1}$. We display an injection of $\mathbb{F}$-algebras $\psi: \Delta_{q} \rightarrow \hat{H}_{q}$ that sends

$$
A \mapsto t_{1} t_{0}+\left(t_{1} t_{0}\right)^{-1}, \quad B \mapsto t_{3} t_{0}+\left(t_{3} t_{0}\right)^{-1}, \quad C \mapsto t_{2} t_{0}+\left(t_{2} t_{0}\right)^{-1} .
$$

The map $\psi$ sends

$$
\begin{aligned}
& \alpha \mapsto\left(q^{-1} t_{0}+q t_{0}^{-1}\right)\left(t_{1}+t_{1}^{-1}\right)+\left(t_{2}+t_{2}^{-1}\right)\left(t_{3}+t_{3}^{-1}\right), \\
& \beta \mapsto\left(q^{-1} t_{0}+q t_{0}^{-1}\right)\left(t_{3}+t_{3}^{-1}\right)+\left(t_{1}+t_{1}^{-1}\right)\left(t_{2}+t_{2}^{-1}\right), \\
& \gamma \mapsto\left(q^{-1} t_{0}+q t_{0}^{-1}\right)\left(t_{2}+t_{2}^{-1}\right)+\left(t_{3}+t_{3}^{-1}\right)\left(t_{1}+t_{1}^{-1}\right) .
\end{aligned}
$$

We show that $\psi$ sends $\Omega$ to

$$
\begin{gathered}
\left(q+q^{-1}\right)^{2}-\left(q^{-1} t_{0}+q t_{0}^{-1}\right)^{2}-\left(t_{1}+t_{1}^{-1}\right)^{2}-\left(t_{2}+t_{2}^{-1}\right)^{2}-\left(t_{3}+t_{3}^{-1}\right)^{2} \\
-\left(q^{-1} t_{0}+q t_{0}^{-1}\right)\left(t_{1}+t_{1}^{-1}\right)\left(t_{2}+t_{2}^{-1}\right)\left(t_{3}+t_{3}^{-1}\right) .
\end{gathered}
$$

We remark that for the above results some parts are easier to prove than others. It is relatively easy to show that $\psi$ exists as an algebra homomorphism. Indeed this existence essentially follows from [7, Theorem 5.2], although in our formal argument we take another approach which quickly yields the result from first principles. We found it relatively hard to show that $\psi$ is injective; indeed this argument takes up the majority of the paper. To establish injectivity we display a basis for $\hat{H}_{q}$, and use it to show that $\psi$ sends the basis (1.2) to a linearly independent set. Adapting [5, Theorem 2.6], [7, Lemma 4.2] we show how the Artin braid group $B_{3}$ acts on $\hat{H}_{q}$ as a group of automorphisms. The group $B_{3}$ is a homomorphic preimage of $\mathrm{PSL}_{2}(\mathbb{Z})$, and we mentioned earlier that $\mathrm{PSL}_{2}(\mathbb{Z})$ acts on $\Delta_{q}$ as a group of automorphisms. Pulling back this $\mathrm{PSL}_{2}(\mathbb{Z})$ action we get a $B_{3}$ action on $\Delta_{q}$ as a group of automorphisms. We show that $\psi$ commutes with the $B_{3}$ actions for $\Delta_{q}$ and $\hat{H}_{q}$. Now consider the image of $\Delta_{q}$ under $\psi$. Adapting [11, Theorem 5.1] we show that the subalgebra $\left\{h \in \hat{H}_{q} \mid t_{0} h=h t_{0}\right\}$ is generated by this image together with $t_{0}$ and $\left\{t_{i}+t_{i}^{-1}\right\}_{i=1}^{3}$. For this subalgebra we give a presentation by generators and relations. Roughly speaking, this presentation amounts to a $q$-analog of [13, Theorem 2.1] and a universal analog of [10, Definition 6.1, Corollary 6.3]. Under the assumption that $q$ is not a root of unity, we show that $Z\left(\hat{H}_{q}\right)$ is generated by $\left\{t_{i}+t_{i}^{-1}\right\}_{i=0}^{3}$ and that $Z\left(\hat{H}_{q}\right)$ is isomorphic to a polynomial algebra in four variables. Roughly speaking, this is a universal analog of [11, Theorem 5.3].

The paper is organized as follows. In Section 2, after reviewing $\Delta_{q}$ we obtain a basis for this algebra that will be useful. In Section 3 we define $\hat{H}_{q}$ and discuss its symmetries. In Section 4 we state five theorems which describe an injection $\psi: \Delta_{q} \rightarrow \hat{H}_{q}$; these are Theorems 4.1-4.5. In Section 5 we establish some identities in $\hat{H}_{q}$ that will be used repeatedly. In Section 6 we prove Theorems 4.1, 4.2, 4.3. In Section 7 we display a basis for $\hat{H}_{q}$, along with some reduction rules that show how to write any given element of $\hat{H}_{q}$ in the basis. Sections 8,9 are devoted to proving Theorem 4.4. Sections 10-12 are devoted to proving Theorem 4.5. In Section 13 we consider the image of $\Delta_{q}$ under the map $\psi$. We show that the subalgebra $\left\{h \in \hat{H}_{q} \mid t_{0} h=h t_{0}\right\}$ is generated by this image together with $t_{0}$ and $\left\{t_{i}+t_{i}^{-1}\right\}_{i=1}^{3}$. In Section 14 we give a presentation for this subalgebra by generators and relations. In Section 15 we describe $Z\left(\hat{H}_{q}\right)$. In Section 16 we compare our results with those of Koornwinder [10, 11]. 


\section{The universal Askey-Wilson algebra}

We now begin our formal argument. In this section we discuss the universal Askey-Wilson algebra. After reviewing its basic features we establish a basis for the algebra that will be useful later in the paper.

Definition 2.1 ([15, Definition 1.2]). Define an $\mathbb{F}$-algebra $\Delta_{q}$ by generators and relations in the following way. The generators are $A, B, C$. The relations assert that each of

$$
A+\frac{q B C-q^{-1} C B}{q^{2}-q^{-2}}, \quad B+\frac{q C A-q^{-1} A C}{q^{2}-q^{-2}}, \quad C+\frac{q A B-q^{-1} B A}{q^{2}-q^{-2}}
$$

is central in $\Delta_{q}$. The algebra $\Delta_{q}$ is called the universal Askey-Wilson algebra.

Definition 2.2 ([15, Definition 1.3]). For the three central elements in (2.1), multiply each by $q+q^{-1}$ to get $\alpha, \beta, \gamma$. Thus

$$
\begin{aligned}
& A+\frac{q B C-q^{-1} C B}{q^{2}-q^{-2}}=\frac{\alpha}{q+q^{-1}}, \\
& B+\frac{q C A-q^{-1} A C}{q^{2}-q^{-2}}=\frac{\beta}{q+q^{-1}}, \\
& C+\frac{q A B-q^{-1} B A}{q^{2}-q^{-2}}=\frac{\gamma}{q+q^{-1}} .
\end{aligned}
$$

Note that each of $\alpha, \beta, \gamma$ is central in $\Delta_{q}$. Note also that $A, B, \gamma$ is a generating set for $\Delta_{q}$.

We now discuss some automorphisms of $\Delta_{q}$. Recall that the modular group $\operatorname{PSL}_{2}(\mathbb{Z})$ has a presentation by generators $p, s$ and relations $p^{3}=1, s^{2}=1$. See for example [1].

Lemma 2.3 ([15, Theorem 3.1]). The group $\mathrm{PSL}_{2}(\mathbb{Z})$ acts on $\Delta_{q}$ as a group of automorphisms in the following way:

\begin{tabular}{c|ccc|ccc}
$u$ & $A$ & $B$ & $C$ & $\alpha$ & $\beta$ & $\gamma$ \\
\hline$p(u)$ & $B$ & $C$ & $A$ & $\beta$ & $\gamma$ & $\alpha$ \\
$s(u)$ & $B$ & $A$ & $C+\frac{A B-B A}{q-q^{-1}}$ & $\beta$ & $\alpha$ & $\gamma$
\end{tabular}

The group $\mathrm{PSL}_{2}(\mathbb{Z})$ has a central extension called the Artin braid group $B_{3}$. The group $B_{3}$ is defined as follows.

Definition 2.4. The Artin braid group $B_{3}$ is defined by generators $\rho, \sigma$ and relations $\rho^{3}=\sigma^{2}$. For notational convenience define $\tau=\rho^{3}=\sigma^{2}$.

There exists a group homomorphism $B_{3} \rightarrow \operatorname{PSL}_{2}(\mathbb{Z})$ that sends $\rho \mapsto p$ and $\sigma \mapsto s$. Via this homomorphism we pull back the $\mathrm{PSL}_{2}(\mathbb{Z})$ action on $\Delta_{q}$, to get a $B_{3}$ action on $\Delta_{q}$ as a group of automorphisms. This action is described as follows.

Lemma 2.5. The group $B_{3}$ acts on $\Delta_{q}$ as a group of automorphisms such that $\tau(h)=h$ for all $h \in \Delta_{q}$ and $\rho, \sigma$ do the following:

\begin{tabular}{c|ccc|ccc}
$u$ & $A$ & $B$ & $C$ & $\alpha$ & $\beta$ & $\gamma$ \\
\hline$\rho(u)$ & $B$ & $C$ & $A$ & $\beta$ & $\gamma$ & $\alpha$ \\
$\sigma(u)$ & $B$ & $A$ & $C+\frac{A B-B A}{q-q^{-1}}$ & $\beta$ & $\alpha$ & $\gamma$
\end{tabular}

In Definition 2.2 we defined the central elements $\alpha, \beta, \gamma$ of $\Delta_{q}$. There is another central element of interest, called the Casimir element $\Omega$. This element is defined as follows. 
Definition 2.6 ([15, Lemma 6.1]). Define an element $\Omega \in \Delta_{q}$ by

$$
\Omega=q^{-1} A C B+q^{-2} A^{2}+q^{-2} B^{2}+q^{2} C^{2}-q^{-1} A \alpha-q^{-1} B \beta-q C \gamma .
$$

We call $\Omega$ the Casimir element of $\Delta_{q}$.

Lemma 2.7 ([15, Theorems 6.2, 8.2]). The Casimir element $\Omega$ is contained in the center $Z\left(\Delta_{q}\right)$. Moreover $\left\{\Omega^{i} \alpha^{r} \beta^{s} \gamma^{t} \mid i, r, s, t \in \mathbb{N}\right\}$ is a basis for the $\mathbb{F}$-vector space $Z\left(\Delta_{q}\right)$, provided that $q$ is not a root of unity.

Lemma 2.8 ([15, Theorem 6.4]). The Casimir element $\Omega$ is fixed by everything in $B_{3}$.

Given an $\mathbb{F}$-algebra $\mathcal{A}$, by an antiautomorphism of $\mathcal{A}$ we mean an $\mathbb{F}$-linear bijection $\zeta: \mathcal{A} \rightarrow \mathcal{A}$ such that $(u v)^{\zeta}=v^{\zeta} u^{\zeta}$ for all $u, v \in \mathcal{A}$.

Lemma 2.9. There exists an antiautomorphism $\dagger$ of $\Delta_{q}$ that sends

$$
A \mapsto B, \quad B \mapsto A, \quad C \mapsto C, \quad \alpha \mapsto \beta, \quad \beta \mapsto \alpha, \quad \gamma \mapsto \gamma
$$

Moreover $\dagger^{2}=1$.

Proof. Routine using (2.2)-(2.4).

Lemma 2.10. The Casimir element $\Omega$ is fixed by the antiautomorphism $\dagger$ from Lemma 2.9.

Proof. This follows from [15, Lemma 6.1].

We mention how $\Delta_{q}$ and $\Delta_{q^{-1}}$ are related.

Lemma 2.11. There exists an isomorphism of $\mathbb{F}$-algebras $\xi: \Delta_{q} \rightarrow \Delta_{q^{-1}}$ that sends

$$
A \mapsto B, \quad B \mapsto A, \quad C \mapsto C, \quad \alpha \mapsto \beta, \quad \beta \mapsto \alpha, \quad \gamma \mapsto \gamma .
$$

Proof. Routine using (2.2)-(2.4).

Lemma 2.12. The isomorphism $\xi: \Delta_{q} \rightarrow \Delta_{q^{-1}}$ from Lemma 2.11 sends the Casimir element of $\Delta_{q}$ to the Casimir element of $\Delta_{q^{-1}}$.

Proof. This follows from [15, Lemma 6.1].

We are going to display a basis for the $\mathbb{F}$-vector space $\Delta_{q}$. Two such bases can be found in $[15$, Theorems $4.1,7.5]$, but these are not suited for our present purpose. To obtain a suitable basis we work with the following presentation of $\Delta_{q}$.

Proposition 2.13. The $\mathbb{F}$-algebra $\Delta_{q}$ is presented by generators and relations in the following way. The generators are $A, B, C, \Omega, \alpha, \beta, \gamma$. The relations assert that each of $\Omega, \alpha, \beta, \gamma$ is central and

$$
\begin{aligned}
& B A=q^{2} A B+q\left(q^{2}-q^{-2}\right) C-q\left(q-q^{-1}\right) \gamma, \\
& B C=q^{-2} C B-q^{-1}\left(q^{2}-q^{-2}\right) A+q^{-1}\left(q-q^{-1}\right) \alpha, \\
& C A=q^{-2} A C-q^{-1}\left(q^{2}-q^{-2}\right) B+q^{-1}\left(q-q^{-1}\right) \beta, \\
& C^{2}=q^{-2} \Omega-q^{-3} A C B-q^{-4} A^{2}-q^{-4} B^{2}+q^{-3} A \alpha+q^{-3} B \beta+q^{-1} C \gamma .
\end{aligned}
$$

Proof. Referring to the above four equations, the first three are reformulations of (2.2)-(2.4) and the fourth is a reformulation of (2.5). 
Definition 2.14. The generators $A, B, C, \Omega, \alpha, \beta, \gamma$ of $\Delta_{q}$ are called balanced.

Note 2.15. Referring to the presentation of $\Delta_{q}$ from Proposition 2.13, consider the relations which assert that $\Omega, \alpha, \beta, \gamma$ are central. These relations can be expressed as

$$
\begin{aligned}
& \Omega A=A \Omega, \quad \Omega B=B \Omega, \quad \Omega C=C \Omega, \\
& \alpha A=A \alpha, \quad \alpha B=B \alpha, \quad \alpha C=C \alpha, \\
& \beta A=A \beta, \quad \beta B=B \beta, \quad \beta C=C \beta, \\
& \gamma A=A \gamma, \quad \gamma B=B \gamma, \quad \gamma C=C \gamma, \\
& \alpha \Omega=\Omega \alpha, \quad \beta \Omega=\Omega \beta, \quad \gamma \Omega=\Omega \gamma, \\
& \beta \alpha=\alpha \beta, \quad \gamma \alpha=\alpha \gamma, \quad \gamma \beta=\beta \gamma .
\end{aligned}
$$

Definition 2.16. By a reduction rule for $\Delta_{q}$ we mean an equation which appears in Proposition 2.13 or Note 2.15. A reduction rule from Proposition 2.13 is said to be of the first kind. A reduction rule from Note 2.15 is said to be of the second kind.

Definition 2.17. For an integer $n \geq 0$, by a word of length $n$ in $\Delta_{q}$ we mean a product $g_{1} g_{2} \cdots g_{n}$ such that $g_{i}$ is a balanced generator of $\Delta_{q}$ for $1 \leq i \leq n$. We interpret the word of length 0 as the multiplicative identity in $\Delta_{q}$. A word is called forbidden whenever it is the left-hand side of a reduction rule. Every forbidden word has length two. A forbidden word is said to be of the first kind (resp. second kind) whenever the corresponding reduction rule is of the first (resp. second) kind.

Definition 2.18. Let $w$ denote a forbidden word in $\Delta_{q}$, and consider the corresponding reduction rule. By a descendent of $w$ we mean a word that appears on the right-hand side of that reduction rule.

Example 2.19. The descendents of $B A$ are $A B, C, \gamma$. The descendents of $B C$ are $C B, A, \alpha$. The descendents of $C A$ are $A C, B, \beta$. The descendents of $C^{2}$ are $\Omega, A C B, A^{2}, B^{2}, A \alpha, B \beta, C \gamma$. A forbidden word of the second kind has a single descendent, obtained by interchanging its two factors.

Theorem 2.20. The following is a basis for the $\mathbb{F}$-vector space $\Delta_{q}$ :

$$
A^{i} C^{j} B^{k} \Omega^{\ell} \alpha^{r} \beta^{s} \gamma^{t}, \quad j \in\{0,1\}, \quad i, k, \ell, r, s, t \in \mathbb{N} .
$$

Proof. We invoke Bergman's Diamond Lemma [3, Theorem 1.2]. Let $g_{1} g_{2} \cdots g_{n}$ denote a word in $\Delta_{q}$. This word is called reducible whenever there exists an integer $i(2 \leq i \leq n)$ such that $g_{i-1} g_{i}$ is forbidden. The word is called irreducible whenever it is not reducible. The list (2.6) consists of the irreducible words in $\Delta_{q}$. Let $w=g_{1} g_{2} \cdots g_{n}$ denote a word in $\Delta_{q}$. By an inversion in $w$ we mean an ordered pair of integers $(i, j)$ such that $1 \leq i<j \leq n$ and the word $g_{i} g_{j}$ is forbidden. The inversion $(i, j)$ is of the first kind (resp. second kind) whenever the forbidden word $g_{i} g_{j}$ is of the first kind (resp. second kind). Let $W$ denote the set of all words in $\Delta_{q}$. We define a partial order $<$ on $W$ as follows. Pick any words $w, w^{\prime}$ in $W$ and write $w=g_{1} g_{2} \cdots g_{n}$. We say that $w$ dominates $w^{\prime}$ whenever there exists an integer $i(2 \leq i \leq n)$ such that $(i-1, i)$ is an inversion for $w$, and $w^{\prime}$ is obtained from $w$ by replacing $g_{i-1} g_{i}$ by one of its descendents. In this case either (i) $w$ has more inversions of the first kind than $w^{\prime}$, or (ii) $w$ and $w^{\prime}$ have the same number of inversions of the first kind, but $w$ has more inversions of the second kind than $w^{\prime}$. By these comments the transitive closure of the domination relation on $W$ is a partial order on $W$ which we denote by $<$. By construction $<$ is a semigroup partial order [3, p. 181] and satisfies the descending chain condition [3, p. 179]. We now relate the partial order $<$ to our reduction rules. Let $w=g_{1} g_{2} \cdots g_{n}$ denote a reducible word in $\Delta_{q}$. Then there exists an 
integer $i(2 \leq i \leq n)$ such that $g_{i-1} g_{i}$ is forbidden. There exists a reduction rule with $g_{i-1} g_{i}$ on the left-hand side; in $w$ we eliminate $g_{i-1} g_{i}$ using this reduction rule and thereby express $w$ as a linear combination of words, each less than $w$ with respect to $<$. Therefore the reduction rules are compatible with $<$ in the sense of Bergman [3, p. 181]. In order to employ the Diamond Lemma, we must show that the ambiguities are resolvable in the sense of Bergman [3, p. 181]. There are potentially two kinds of ambiguities; inclusion ambiguities and overlap ambiguities [3, p. 181]. For the present example there are no inclusion ambiguities. The nontrivial overlap ambiguities are

$$
B C A, \quad B C^{2}, \quad C^{2} A .
$$

Take for example $B C A$. The words $B C$ and $C A$ are forbidden. Therefore $B C A$ can be reduced in two ways; we could evaluate $B C$ first or we could evaluate $C A$ first. Either way, after a 4-step reduction we get the same resolution, which is

$$
\begin{gathered}
q^{-3}\left(q^{2}-q^{-2}\right) \Omega+q^{-6} A C B-q^{-3}\left(q^{4}-q^{-4}\right) A^{2}-q^{-3}\left(q^{4}-q^{-4}\right) B^{2} \\
+q^{-3}\left(q^{3}-q^{-3}\right) A \alpha+q^{-3}\left(q^{3}-q^{-3}\right) B \beta+q^{-3}\left(q-q^{-1}\right) C \gamma
\end{gathered}
$$

Therefore the ambiguity $B C A$ is resolvable. The ambiguities $B C^{2}$ and $C^{2} A$ are similarly shown to be resolvable. The resolution of $B C^{2}$ is

$$
\begin{array}{rl}
q^{-6} & B \Omega-q^{-7} A C B^{2}-q^{-8} A^{2} B-q^{-8} B^{3}+q^{-7} A B \alpha+q^{-7} B^{2} \beta+q^{-5} C B \gamma \\
& -q^{-3}\left(q^{4}-q^{-4}\right) A C+q^{-2}\left(q^{2}-q^{-2}\right) C \alpha+q^{-4}\left(q^{2}-q^{-2}\right)^{2} B \\
& -q^{-4}\left(q-q^{-1}\right)\left(q^{2}-q^{-2}\right) \beta
\end{array}
$$

and the resolution of $C^{2} A$ is

$$
\begin{aligned}
q^{-6} A & \Omega-q^{-7} A^{2} C B-q^{-8} A B^{2}-q^{-8} A^{3}+q^{-7} A^{2} \alpha+q^{-7} A B \beta+q^{-5} A C \gamma \\
& -q^{-3}\left(q^{4}-q^{-4}\right) C B+q^{-2}\left(q^{2}-q^{-2}\right) C \beta+q^{-4}\left(q^{2}-q^{-2}\right)^{2} A \\
& -q^{-4}\left(q-q^{-1}\right)\left(q^{2}-q^{-2}\right) \alpha .
\end{aligned}
$$

We conclude that every ambiguity is resolvable, so by the Diamond Lemma [3, Theorem 1.2] the irreducible words form a basis for $\Delta_{q}$. The result follows.

\section{The universal DAHA $\hat{H}_{q}$ of type $\left(C_{1}^{\vee}, C_{1}\right)$}

The double affine Hecke algebra (DAHA) for a reduced root system was defined by Cherednik [4], and the definition was extended to include nonreduced root systems by Sahi [14]. The most general DAHA of rank 1 is associated with the root system $\left(C_{1}^{\vee}, C_{1}\right)$. In [7] we introduced a central extension of this algebra called the universal DAHA of type $\left(C_{1}^{\vee}, C_{1}\right)$. In the present paper we will work with a variation on this algebra.

For notational convenience define a four element set

$$
\mathbb{I}=\{0,1,2,3\} .
$$

The following definition is a variation on [7, Definition 3.1].

Definition 3.1. Let $\hat{H}_{q}$ denote the $\mathbb{F}$-algebra defined by generators $\left\{t_{i}^{ \pm 1}\right\}_{i \in \mathbb{I}}$ and relations

$$
\begin{aligned}
& t_{i} t_{i}^{-1}=t_{i}^{-1} t_{i}=1, \quad i \in \mathbb{I}, \\
& t_{i}+t_{i}^{-1} \text { is central, } \quad i \in \mathbb{I}, \\
& t_{0} t_{1} t_{2} t_{3}=q^{-1} .
\end{aligned}
$$

We call $\hat{H}_{q}$ the universal DAHA of type $\left(C_{1}^{\vee}, C_{1}\right)$. 
Remark 3.2. In [7, Definition 3.1] we defined an $\mathbb{F}$-algebra $\hat{H}$ by generators $\left\{t_{i}^{ \pm 1}\right\}_{i \in \mathbb{I}}$ and relations (i) $t_{i} t_{i}^{-1}=t_{i}^{-1} t_{i}=1$; (ii) $t_{i}+t_{i}^{-1}$ is central; (iii) $t_{0} t_{1} t_{2} t_{3}$ is central. The algebra $\hat{H}_{q}$ is a homomorphic image of $\hat{H}$.

The following two lemmas are immediate from Definition 3.1.

Lemma 3.3. In the algebra $\hat{H}_{q}$ the scalar $q^{-1}$ is equal to each of the following:

$$
t_{0} t_{1} t_{2} t_{3}, \quad t_{1} t_{2} t_{3} t_{0}, \quad t_{2} t_{3} t_{0} t_{1}, \quad t_{3} t_{0} t_{1} t_{2} .
$$

Lemma 3.4. There exists an automorphism of $\hat{H}_{q}$ that sends $t_{0} \mapsto t_{1} \mapsto t_{2} \mapsto t_{3} \mapsto t_{0}$.

Recall the braid group $B_{3}$ from Definition 2.4.

Lemma 3.5. The group $B_{3}$ acts on $\hat{H}_{q}$ as a group of automorphisms such that $\tau(h)=t_{0}^{-1} h t_{0}$ for all $h \in \hat{H}_{q}$ and $\rho, \sigma$ do the following:

\begin{tabular}{c|cccc}
$h$ & $t_{0}$ & $t_{1}$ & $t_{2}$ & $t_{3}$ \\
\hline$\rho(h)$ & $t_{0}$ & $t_{0}^{-1} t_{3} t_{0}$ & $t_{1}$ & $t_{2}$ \\
$\sigma(h)$ & $t_{0}$ & $t_{0}^{-1} t_{3} t_{0}$ & $t_{1} t_{2} t_{1}^{-1}$ & $t_{1}$
\end{tabular}

Proof. This is routinely checked, or see [7, Lemma 4.2].

Lemma 3.6. The $B_{3}$ action on $\hat{H}_{q}$ does the following to the central elements (3.1). The generator $\tau$ fixes every central element. The generators $\rho, \sigma$ satisfy the table below.

\begin{tabular}{c|cccc}
$h$ & $t_{0}+t_{0}^{-1}$ & $t_{1}+t_{1}^{-1}$ & $t_{2}+t_{2}^{-1}$ & $t_{3}+t_{3}^{-1}$ \\
\hline$\rho(h)$ & $t_{0}+t_{0}^{-1}$ & $t_{3}+t_{3}^{-1}$ & $t_{1}+t_{1}^{-1}$ & $t_{2}+t_{2}^{-1}$ \\
$\sigma(h)$ & $t_{0}+t_{0}^{-1}$ & $t_{3}+t_{3}^{-1}$ & $t_{2}+t_{2}^{-1}$ & $t_{1}+t_{1}^{-1}$
\end{tabular}

Proof. Use (3.1) and Lemma 3.5.

Lemma 3.7. There exists a unique antiautomorphism $\dagger$ of $\hat{H}_{q}$ that sends

$$
t_{0} \mapsto t_{0}, \quad t_{1} \mapsto t_{3}, \quad t_{2} \mapsto t_{2}, \quad t_{3} \mapsto t_{1}
$$

Moreover $\dagger^{2}=1$.

Proof. Use Definition 3.1.

Lemma 3.8. There exists a unique isomorphism of $\mathbb{F}$-algebras $\xi: \hat{H}_{q} \rightarrow \hat{H}_{q^{-1}}$ that sends

$$
t_{0} \mapsto t_{0}^{-1}, \quad t_{1} \mapsto t_{3}^{-1}, \quad t_{2} \mapsto t_{2}^{-1}, \quad t_{3} \mapsto t_{1}^{-1}
$$

Proof. Use Definition 3.1.

\section{How $\Delta_{q}$ is related to $\hat{H}_{q}$}

In this section we state five theorems concerning how $\Delta_{q}$ is related to $\hat{H}_{q}$. The proofs of these theorems will take up most of the rest of the paper.

Theorem 4.1. There exists a unique $\mathbb{F}$-algebra homomorphism $\psi: \Delta_{q} \rightarrow \hat{H}_{q}$ that sends

$$
A \mapsto t_{1} t_{0}+\left(t_{1} t_{0}\right)^{-1}, \quad B \mapsto t_{3} t_{0}+\left(t_{3} t_{0}\right)^{-1}, \quad C \mapsto t_{2} t_{0}+\left(t_{2} t_{0}\right)^{-1} .
$$

The homomorphism $\psi$ sends

$$
\begin{aligned}
& \alpha \mapsto\left(q^{-1} t_{0}+q t_{0}^{-1}\right)\left(t_{1}+t_{1}^{-1}\right)+\left(t_{2}+t_{2}^{-1}\right)\left(t_{3}+t_{3}^{-1}\right), \\
& \beta \mapsto\left(q^{-1} t_{0}+q t_{0}^{-1}\right)\left(t_{3}+t_{3}^{-1}\right)+\left(t_{1}+t_{1}^{-1}\right)\left(t_{2}+t_{2}^{-1}\right), \\
& \gamma \mapsto\left(q^{-1} t_{0}+q t_{0}^{-1}\right)\left(t_{2}+t_{2}^{-1}\right)+\left(t_{3}+t_{3}^{-1}\right)\left(t_{1}+t_{1}^{-1}\right) .
\end{aligned}
$$


Theorem 4.2. For all $g \in B_{3}$ the following diagram commutes:

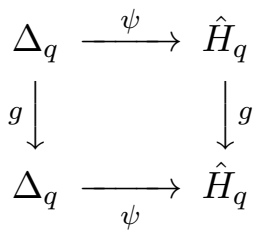

Theorem 4.3. The following diagrams commute:

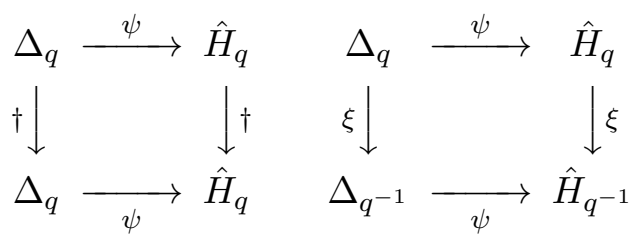

Theorem 4.4. Under the homomorphism $\psi$ from Theorem 4.1 the image of $\Omega$ is

$$
\begin{aligned}
& \left(q+q^{-1}\right)^{2}-\left(q^{-1} t_{0}+q t_{0}^{-1}\right)^{2}-\left(t_{1}+t_{1}^{-1}\right)^{2}-\left(t_{2}+t_{2}^{-1}\right)^{2}-\left(t_{3}+t_{3}^{-1}\right)^{2} \\
& \quad-\left(q^{-1} t_{0}+q t_{0}^{-1}\right)\left(t_{1}+t_{1}^{-1}\right)\left(t_{2}+t_{2}^{-1}\right)\left(t_{3}+t_{3}^{-1}\right) .
\end{aligned}
$$

Theorem 4.5. The homomorphism $\psi$ from Theorem 4.1 is injective.

\section{Preliminaries concerning $\hat{\boldsymbol{H}}_{q}$}

In this section we establish some basic facts about $\hat{H}_{q}$. These facts will be used repeatedly for the rest of the paper.

Definition 5.1. For the algebra $\hat{H}_{q}$ define

$$
T_{i}=t_{i}+t_{i}^{-1}, \quad i \in \mathbb{I} .
$$

Note that each $T_{i}$ is central in $\hat{H}_{q}$.

In Definition 3.1 we gave a presentation for $\hat{H}_{q}$ involving the generators $\left\{t_{i}^{ \pm 1}\right\}_{i \in \mathbb{I}}$. Sometimes it is convenient to use $\left\{T_{i}\right\}_{i \in \mathbb{I}}$ instead of $\left\{t_{i}^{-1}\right\}_{i \in \mathbb{I}}$. In terms of the generators $\left\{t_{i}\right\}_{i \in \mathbb{I}},\left\{T_{i}\right\}_{i \in \mathbb{I}}$ the algebra $\hat{H}_{q}$ looks as follows.

Lemma 5.2. The $\mathbb{F}$-algebra $\hat{H}_{q}$ has a presentation by generators $\left\{t_{i}\right\}_{i \in \mathbb{I}},\left\{T_{i}\right\}_{i \in \mathbb{I}}$ and relations

$$
\begin{aligned}
& t_{i}^{2}=T_{i} t_{i}-1, \quad i \in \mathbb{I}, \\
& T_{i} \text { is central, } \quad i \in \mathbb{I}, \\
& t_{0} t_{1} t_{2} t_{3}=q^{-1} .
\end{aligned}
$$

Definition 5.3. Let $X, Y$ denote the following elements of $\hat{H}_{q}$ :

$$
X=t_{3} t_{0}, \quad Y=t_{0} t_{1} .
$$

Note that each of $X, Y$ is invertible.

Lemma 5.4. For the algebra $\hat{H}_{q}$,

$$
t_{1}=t_{0}^{-1} Y, \quad t_{2}=q^{-1} Y^{-1} t_{0} X^{-1}, \quad t_{3}=X t_{0}^{-1} .
$$

Moreover $\hat{H}_{q}$ is generated by $X^{ \pm 1}, Y^{ \pm 1}, t_{0}^{ \pm 1}$. 
Proof. The relations (5.3) are routinely checked using Definition 3.1 and (5.2).

In terms of the generators $X^{ \pm 1}, Y^{ \pm 1}, t_{0}^{ \pm 1}$ the $\left\{T_{i}\right\}_{i \in \mathbb{I}}$ look as follows.

Lemma 5.5. For the algebra $\hat{H}_{q}$ the following $(i)-(i v)$ hold.

(i) $T_{0}=t_{0}+t_{0}^{-1}$.

(ii) $T_{1}$ is equal to each of

$$
t_{0}^{-1} Y+Y^{-1} t_{0}, \quad Y t_{0}^{-1}+t_{0} Y^{-1}
$$

(iii) $T_{2}$ is equal to each of

$$
\begin{aligned}
& q t_{0}^{-1} Y X+q^{-1} X^{-1} Y^{-1} t_{0}, \quad q X t_{0}^{-1} Y+q^{-1} Y^{-1} t_{0} X^{-1} \\
& q Y X t_{0}^{-1}+q^{-1} t_{0} X^{-1} Y^{-1} .
\end{aligned}
$$

(iv) $T_{3}$ is equal to each of

$$
t_{0}^{-1} X+X^{-1} t_{0}, \quad X t_{0}^{-1}+t_{0} X^{-1} .
$$

Proof. (i) Clear.

(ii) Using the equation on the left in (5.3),

$$
T_{1}=t_{1}+t_{1}^{-1}=t_{0}^{-1} Y+Y^{-1} t_{0} .
$$

Also

$$
T_{1}=Y T_{1} Y^{-1}=Y\left(t_{0}^{-1} Y+Y^{-1} t_{0}\right) Y^{-1}=Y t_{0}^{-1}+t_{0} Y^{-1} .
$$

(iii) Using the middle equation in (5.3),

$$
T_{2}=t_{2}^{-1}+t_{2}=q X t_{0}^{-1} Y+q^{-1} Y^{-1} t_{0} X^{-1} .
$$

Also

$$
T_{2}=X^{-1} T_{2} X=X^{-1}\left(q X t_{0}^{-1} Y+q^{-1} Y^{-1} t_{0} X^{-1}\right) X=q t_{0}^{-1} Y X+q^{-1} X^{-1} Y^{-1} t_{0}
$$

and

$$
T_{2}=Y T_{2} Y^{-1}=Y\left(q X t_{0}^{-1} Y+q^{-1} Y^{-1} t_{0} X^{-1}\right) Y^{-1}=q Y X t_{0}^{-1}+q^{-1} t_{0} X^{-1} Y^{-1} .
$$

(iv) Similar to the proof of $(i i)$ above.

In Section 3 we discussed some automorphisms and antiautomorphisms of $\hat{H}_{q}$. We now consider how these maps act on $X, Y$. The following four lemmas are routinely checked.

Lemma 5.6. Consider the automorphism of $\hat{H}_{q}$ from Lemma 3.4. This automorphism sends

$$
X \mapsto Y \mapsto q^{-1} X^{-1} \mapsto q^{-1} Y^{-1} \mapsto X
$$

Lemma 5.7. Consider the automorphisms $\rho, \sigma$ of $\hat{H}_{q}$ from Lemma 3.5. The automorhism $\rho$ sends

$$
X \mapsto q^{-1} Y^{-1} t_{0} X^{-1} t_{0}, \quad Y \mapsto X .
$$

The automorphism $\sigma$ sends

$$
X \mapsto t_{0}^{-1} Y t_{0}, \quad Y \mapsto X .
$$


Lemma 5.8. Recall the antiautomorphism $\dagger$ of $\hat{H}_{q}$ from Lemma 3.7. This map swaps $X, Y$.

Lemma 5.9. Recall the isomorphism $\xi: \hat{H}_{q} \rightarrow \hat{H}_{q^{-1}}$ from Lemma 3.8. This map sends $X \mapsto$ $Y^{-1}$ and $Y \mapsto X^{-1}$.

We now give some relations that show how $t_{0}$ commutes past the $X^{ \pm 1}, Y^{ \pm 1}$.

Lemma 5.10. The following relations hold in $\hat{H}_{q}$ :

$$
\begin{aligned}
& t_{0} X=X^{-1} t_{0}+X T_{0}-T_{3}, \\
& t_{0} X^{-1}=X t_{0}-X T_{0}+T_{3}, \\
& t_{0} Y=Y^{-1} t_{0}+Y T_{0}-T_{1}, \\
& t_{0} Y^{-1}=Y t_{0}-Y T_{0}+T_{1} .
\end{aligned}
$$

Proof. To obtain (5.4), (5.5) replace $t_{0}^{-1}$ by $T_{0}-t_{0}$ in Lemma 5.5(iv). To obtain (5.6), (5.7) replace $t_{0}^{-1}$ by $T_{0}-t_{0}$ in Lemma $5.5(i i)$.

We now consider how $X, Y$ are related.

Lemma 5.11. The following relations hold in $\hat{H}_{q}$ :

$$
\begin{array}{lll}
t_{0} t_{2}=q^{-1} t_{3}^{-1} T_{1}-q^{-1} Y X^{-1}, & t_{0}^{-1} t_{2}^{-1}=q t_{1} T_{3}-q X^{-1} Y, \\
t_{1} t_{3}=q^{-1} t_{0}^{-1} T_{2}-q^{-2} X^{-1} Y^{-1}, & t_{1}^{-1} t_{3}^{-1}=q t_{2} T_{0}-Y^{-1} X^{-1}, \\
t_{2} t_{0}=q^{-1} t_{1}^{-1} T_{3}-q^{-1} Y^{-1} X, & t_{2}^{-1} t_{0}^{-1}=q t_{3} T_{1}-q X Y^{-1}, \\
t_{3} t_{1}=q^{-1} t_{2}^{-1} T_{0}-X Y, & t_{3}^{-1} t_{1}^{-1}=q t_{0} T_{2}-q^{2} Y X .
\end{array}
$$

Proof. Concerning (5.8), the equation on the left comes from

$$
q^{-1} Y X^{-1}=t_{0} t_{1}^{2} t_{2}=t_{0}\left(T_{1} t_{1}-1\right) t_{2}=q^{-1} t_{3}^{-1} T_{1}-t_{0} t_{2} .
$$

The equation on the right comes from

$$
q X^{-1} Y=t_{0}^{-1} t_{3}^{-2} t_{2}^{-1}=t_{0}^{-1}\left(T_{3} t_{3}^{-1}-1\right) t_{2}^{-1}=q t_{1} T_{3}-t_{0}^{-1} t_{2}^{-1} .
$$

To obtain (5.9)-(5.11), repeatedly apply the automorphism from Lemma 3.4 to everything in (5.8), and use Lemma 5.6.

Definition 5.12. Let $\left\{C_{i}\right\}_{i \in \mathbb{I}}$ denote the following elements in $\hat{H}_{q}$ :

$$
\begin{aligned}
& C_{0}=q\left(q Y X-q^{-1} X Y\right) \\
& C_{1}=-\left(q^{-1} Y X^{-1}-q X^{-1} Y\right), \\
& C_{2}=q^{-1}\left(q Y^{-1} X^{-1}-q^{-1} X^{-1} Y^{-1}\right), \\
& C_{3}=-\left(q^{-1} Y^{-1} X-q X Y^{-1}\right) .
\end{aligned}
$$

Lemma 5.13. The automorphism from Lemma 3.4 sends

$$
C_{0} \mapsto C_{1} \mapsto C_{2} \mapsto C_{3} \mapsto C_{0}
$$

Proof. Use Lemma 5.6 and Definition 5.12. 
Proposition 5.14. The following relations hold in $\hat{H}_{q}$ :

$$
\begin{aligned}
& C_{0}=q T_{2} t_{0}+T_{3} t_{1}+q^{-1} T_{0} t_{2}+T_{1} t_{3}-q^{-1} T_{0} T_{2}-T_{1} T_{3}, \\
& C_{1}=T_{2} t_{0}+q T_{3} t_{1}+T_{0} t_{2}+q^{-1} T_{1} t_{3}-T_{0} T_{2}-q^{-1} T_{1} T_{3}, \\
& C_{2}=q^{-1} T_{2} t_{0}+T_{3} t_{1}+q T_{0} t_{2}+T_{1} t_{3}-q^{-1} T_{0} T_{2}-T_{1} T_{3}, \\
& C_{3}=T_{2} t_{0}+q^{-1} T_{3} t_{1}+T_{0} t_{2}+q T_{1} t_{3}-T_{0} T_{2}-q^{-1} T_{1} T_{3} .
\end{aligned}
$$

Proof. To verify (5.12), use (5.11) together with

$$
t_{3}^{-1} t_{1}^{-1}=\left(T_{3}-t_{3}\right)\left(T_{1}-t_{1}\right)=T_{1} T_{3}-t_{3} T_{1}-t_{1} T_{3}+t_{3} t_{1} .
$$

To verify (5.13)-(5.15), repeatedly apply the automorphism from Lemma 3.4 to everything in (5.12), and use Lemma 5.13.

We mention a result for future use.

Lemma 5.15. The automorphism $\sigma$ of $\hat{H}_{q}$ sends

$$
\begin{array}{rlrl}
t_{1} t_{3} & \mapsto q^{-1} t_{0}^{-1} t_{2}^{-1}, & t_{3}^{-1} t_{1}^{-1} \mapsto q t_{2} t_{0}, \\
t_{0} t_{2} \mapsto q^{-1} t_{3}^{-1} t_{1}^{-1}, & t_{2}^{-1} t_{0}^{-1} \mapsto q t_{1} t_{3} .
\end{array}
$$

Proof. This is routinely checked using the action of $\sigma$ given in Lemma 3.5.

\section{The proof of Theorems 4.1, 4.2, 4.3}

In this section we prove the first three theorems from Section 4.

Lemma 6.1 ([7, Lemma 3.8]). Let $u, v$ denote invertible elements in any algebra such that each of $u+u^{-1}, v+v^{-1}$ is central. Then

(i) $u v+(u v)^{-1}=v u+(v u)^{-1}$;

(ii) $u v+(u v)^{-1}$ commutes with each of $u, v$.

Proof. (i) Write $U=u+u^{-1}$ and $V=v+v^{-1}$. We have both

$$
\begin{aligned}
& u v+(u v)^{-1}=u v+(V-v)(U-u)=u v+v u-v U-u V+U V, \\
& v u+(v u)^{-1}=v u+(U-u)(V-v)=v u+u v-u V-v U+U V .
\end{aligned}
$$

The result follows.

(ii) Using (i) we have

$$
u^{-1}\left(u v+v^{-1} u^{-1}\right) u=v u+u^{-1} v^{-1}=u v+v^{-1} u^{-1} .
$$

Therefore $u v+(u v)^{-1}$ commutes with $u$. Similarly $u v+(u v)^{-1}$ commutes with $v$.

Corollary 6.2. For distinct $i, j \in \mathbb{I}$,

(i) $t_{i} t_{j}+\left(t_{i} t_{j}\right)^{-1}=t_{j} t_{i}+\left(t_{j} t_{i}\right)^{-1}$.

(ii) $t_{i} t_{j}+\left(t_{i} t_{j}\right)^{-1}$ commutes with each of $t_{i}, t_{j}$.

Proof. By Lemma 6.1 and since $t_{k}+t_{k}^{-1}$ is central for $k \in \mathbb{I}$. 
Definition 6.3. We define elements $A, B, C$ in $\hat{H}_{q}$ as follows:

$$
\begin{aligned}
& A=t_{1} t_{0}+\left(t_{1} t_{0}\right)^{-1}=t_{0} t_{1}+\left(t_{0} t_{1}\right)^{-1}=Y+Y^{-1}, \\
& B=t_{3} t_{0}+\left(t_{3} t_{0}\right)^{-1}=t_{0} t_{3}+\left(t_{0} t_{3}\right)^{-1}=X+X^{-1}, \\
& C=t_{2} t_{0}+\left(t_{2} t_{0}\right)^{-1}=t_{0} t_{2}+\left(t_{0} t_{2}\right)^{-1} .
\end{aligned}
$$

Lemma 6.4. In the algebra $\hat{H}_{q}$ the element $t_{0}$ commutes with each of $A, B, C$.

Proof. By Corollary 6.2(ii) and Definition 6.3.

The following is a variation on [7, Theorem 5.1].

Lemma 6.5. The $B_{3}$ action on $\hat{H}_{q}$ does the following to the elements $A, B, C$ from Definition 6.3. The generator $\tau$ fixes each of $A, B, C$. The generator $\rho$ sends $A \mapsto B \mapsto C \mapsto A$. The generator $\sigma$ swaps $A, B$ and sends $C \mapsto C^{\prime}$ where

$$
q C+q^{-1} C^{\prime}+A B=q^{-1} C+q C^{\prime}+B A=\left(q^{-1} t_{0}+q t_{0}^{-1}\right) T_{2}+T_{1} T_{3} .
$$

Proof. The generator $\tau$ fixes each of $A, B, C$ by Lemma 6.4 and since $\tau(h)=t_{0}^{-1} h t_{0}$ for all $h \in \hat{H}_{q}$. The generator $\rho$ sends $A \mapsto B \mapsto C \mapsto A$ by Lemma 3.5 and Definition 6.3. Similarly the generator $\sigma$ swaps $A, B$. Define $C^{\prime}=\sigma(C)$. We show that $C^{\prime}$ satisfies the equations of the lemma statement. We first show that

$$
q C+q^{-1} C^{\prime}+A B=\left(q^{-1} t_{0}+q t_{0}^{-1}\right) T_{2}+T_{1} T_{3} .
$$

Since $A=Y+Y^{-1}$ and $B=X+X^{-1}$,

$$
A B=Y X+Y X^{-1}+Y^{-1} X+Y^{-1} X^{-1} .
$$

By (6.1) along with (5.8) and (5.10),

$$
C=t_{0} t_{2}+t_{2}^{-1} t_{0}^{-1}=\left(q t_{3}+q^{-1} t_{3}^{-1}\right) T_{1}-q X Y^{-1}-q^{-1} Y X^{-1} .
$$

Using (6.1) and Lemma 5.15 along with (5.9) and (5.11),

$$
C^{\prime}=q t_{1} t_{3}+q^{-1} t_{3}^{-1} t_{1}^{-1}=T_{0} T_{2}-q Y X-q^{-1} X^{-1} Y^{-1} .
$$

To verify (6.2), evaluate the left-hand side using (6.3)-(6.4) and simplify the result using Definition 5.12, Proposition 5.14, and (5.1). We have verified (6.2). Next we show that

$$
q^{-1} C+q C^{\prime}+B A=\left(q^{-1} t_{0}+q t_{0}^{-1}\right) T_{2}+T_{1} T_{3} .
$$

To obtain (6.5), apply $\sigma$ to each side of (6.2) and evaluate the result. To aid in this evaluation, recall that $\sigma$ swaps $A, B$; also $\sigma$ swaps $C, C^{\prime}$ since $\sigma^{2}=\tau$ and $\tau(C)=C$. By these comments and Lemma 3.6 we routinely obtain (6.5).

The following is a variation on [7, Theorem 5.2].

Proposition 6.6. In the algebra $\hat{H}_{q}$ the elements $A, B, C$ are related as follows:

$$
\begin{aligned}
& A+\frac{q B C-q^{-1} C B}{q^{2}-q^{-2}}=\frac{\left(q^{-1} t_{0}+q t_{0}^{-1}\right) T_{1}+T_{2} T_{3}}{q+q^{-1}}, \\
& B+\frac{q C A-q^{-1} A C}{q^{2}-q^{-2}}=\frac{\left(q^{-1} t_{0}+q t_{0}^{-1}\right) T_{3}+T_{1} T_{2}}{q+q^{-1}}, \\
& C+\frac{q A B-q^{-1} B A}{q^{2}-q^{-2}}=\frac{\left(q^{-1} t_{0}+q t_{0}^{-1}\right) T_{2}+T_{3} T_{1}}{q+q^{-1}} .
\end{aligned}
$$


Proof. To get the last equation, eliminate $C^{\prime}$ from the equations of Lemma 6.5. To get the other two equations use the $B_{3}$ action from Lemma 3.5. Specifically, apply $\rho$ twice to the last equation and use the data in Lemma 3.6, together with the fact that $\rho$ cyclically permutes $A$, $B, C$ and fixes $t_{0}$.

Proof of Theorem 4.1. Immediate from Lemma 6.4 and Proposition 6.6.

Back in Definition 2.2 we defined some elements $\alpha, \beta, \gamma$ of $\Delta_{q}$. From now on we retain the notation $\alpha, \beta, \gamma$ for their images under the map $\psi: \Delta_{q} \rightarrow \hat{H}_{q}$. Thus the elements $\alpha, \beta, \gamma$ of $\hat{H}_{q}$ satisfy

$$
\begin{aligned}
& \alpha=\left(q^{-1} t_{0}+q t_{0}^{-1}\right) T_{1}+T_{2} T_{3}, \\
& \beta=\left(q^{-1} t_{0}+q t_{0}^{-1}\right) T_{3}+T_{1} T_{2}, \\
& \gamma=\left(q^{-1} t_{0}+q t_{0}^{-1}\right) T_{2}+T_{3} T_{1} .
\end{aligned}
$$

Proof of Theorem 4.2. Without loss we may assume $g=\rho$ or $g=\sigma$. By Lemma 2.5 the action of $\rho$ on $\Delta_{q}$ cyclically permutes $A, B, C$. By Lemma 6.5 the action of $\rho$ on $\hat{H}_{q}$ cyclically permutes $A, B, C$. By Lemma 2.5 the action of $\sigma$ on $\Delta_{q}$ swaps $A, B$ and fixes $\gamma$. The action of $\sigma$ on $\hat{H}_{q}$ swaps $A, B$ by Lemma 6.5. The action of $\sigma$ on $\hat{H}_{q}$ fixes $\gamma$ by (6.8) and Lemmas 3.5, 3.6. The result follows.

Proof of Theorem 4.3. In each case, chase $A, B, C$ around the diagram using Theorem 4.1 and Corollary 6.2 $(i)$, together with Lemma 2.9 and 3.7 for $\dagger$ and with Lemma 2.11 and 3.8 for $\xi$.

\section{$7 \quad$ A basis for the $\mathbb{F}$-vector space $\hat{H}_{q}$}

Our next general goal is to prove Theorem 4.4. The proof will be completed in Section 9. In the present section we obtain a basis for the $\mathbb{F}$-vector space $\hat{H}_{q}$. The basis consists of

$$
Y^{i} X^{j} t_{0}^{k} T_{0}^{\ell} T_{1}^{r} T_{2}^{s} T_{3}^{t}, \quad i, j \in \mathbb{Z}, \quad k \in\{0,1\}, \quad \ell, r, s, t \in \mathbb{N} .
$$

We also obtain a set of relations for $\hat{H}_{q}$ called reduction rules. The reduction rules show how to write any given element of $\hat{H}_{q}$ as a linear combination of the basis elements (7.1).

To begin the basis project, we are going to display a presentation of $\hat{H}_{q}$ that contains detailed information about how the generators commute past each other. We will give two versions of this presentation. For version I we attempt to optimize clarity. For version II we attempt to optimize utility. We hope that taken together the two versions are reasonably clear and useful. The relations in version II become our reduction rules.

We now give version $\mathrm{I}$.

Proposition 7.1. The $\mathbb{F}$-algebra $\hat{H}_{q}$ has a presentation by generators $X^{ \pm 1}, Y^{ \pm 1},\left\{t_{i}\right\}_{i \in \mathbb{I}},\left\{T_{i}\right\}_{i \in \mathbb{I}}$, $\left\{C_{i}\right\}_{i \in \mathbb{I}}$ and relations $X X^{-1}=1, X^{-1} X=1, Y Y^{-1}=1, Y^{-1} Y=1$, the $\left\{T_{i}\right\}_{i \in \mathbb{I}}$ are central,

$$
\begin{aligned}
& t_{0}^{2}=t_{0} T_{0}-1, \\
& t_{1}=\left(T_{0}-t_{0}\right) Y, \\
& t_{2}=q^{-1} Y^{-1} t_{0} X^{-1}, \\
& t_{3}=X\left(T_{0}-t_{0}\right), \\
& t_{0} X=X^{-1} t_{0}+X T_{0}-T_{3}, \\
& t_{0} X^{-1}=X t_{0}-X T_{0}+T_{3},
\end{aligned}
$$




$$
\begin{aligned}
& t_{0} Y=Y^{-1} t_{0}+Y T_{0}-T_{1}, \\
& t_{0} Y^{-1}=Y t_{0}-Y T_{0}+T_{1}, \\
& X Y=q^{2} Y X-C_{0}, \\
& X^{-1} Y=q^{-2} Y X^{-1}+q^{-1} C_{1}, \\
& X^{-1} Y^{-1}=q^{2} Y^{-1} X^{-1}-q^{2} C_{2}, \\
& X Y^{-1}=q^{-2} Y^{-1} X+q^{-1} C_{3}, \\
& C_{0}=q T_{2} t_{0}+T_{3} t_{1}+q^{-1} T_{0} t_{2}+T_{1} t_{3}-q^{-1} T_{0} T_{2}-T_{1} T_{3}, \\
& C_{1}=T_{2} t_{0}+q T_{3} t_{1}+T_{0} t_{2}+q^{-1} T_{1} t_{3}-T_{0} T_{2}-q^{-1} T_{1} T_{3}, \\
& C_{2}=q^{-1} T_{2} t_{0}+T_{3} t_{1}+q T_{0} t_{2}+T_{1} t_{3}-q^{-1} T_{0} T_{2}-T_{1} T_{3}, \\
& C_{3}=T_{2} t_{0}+q^{-1} T_{3} t_{1}+T_{0} t_{2}+q T_{1} t_{3}-T_{0} T_{2}-q^{-1} T_{1} T_{3} .
\end{aligned}
$$

Proof. Consider the relations in the proposition statement. We now show that these relations hold in $\hat{H}_{q}$. This is clear for the relations shown in the line, so consider the 16 displayed relations. Displayed relation 1 is from Lemma 5.2. Displayed relations 2-4 follow from Lemma 5.4. Displayed relations 5-8 are from Lemma 5.10. Displayed relations 9-12 are from Definition 5.12. Displayed relations 13-16 are from Proposition 5.14. We have shown that the relations in the proposition statement hold in $\hat{H}_{q}$. Conversely, one routinely checks that the relations in the proposition statement imply the defining relations for $\hat{H}_{q}$ given in Lemma 5.2.

We now give version II. Roughly speaking, this version amounts to a universal analog of [10, Proposition 5.2].

Proposition 7.2. The $\mathbb{F}$-algebra $\hat{H}_{q}$ has a presentation by generators $X^{ \pm 1}, Y^{ \pm 1}, t_{0},\left\{T_{i}\right\}_{i \in \mathbb{I}}$ and relations $X X^{-1}=1, X^{-1} X=1, Y Y^{-1}=1, Y^{-1} Y=1$, the $\left\{T_{i}\right\}_{i \in \mathbb{I}}$ are central,

$$
\begin{aligned}
& t_{0}^{2}=t_{0} T_{0}-1 \\
& t_{0} X=X^{-1} t_{0}+X T_{0}-T_{3} \text {, } \\
& t_{0} X^{-1}=X t_{0}-X T_{0}+T_{3} \text {, } \\
& t_{0} Y=Y^{-1} t_{0}+Y T_{0}-T_{1} \text {, } \\
& t_{0} Y^{-1}=Y t_{0}-Y T_{0}+T_{1} \text {, } \\
& X Y=q^{2} Y X-q t_{0} T_{2}+q^{-1} T_{0} T_{2}+Y^{-1} t_{0} T_{3}-q^{-2} Y^{-1} T_{0} T_{3} \\
& +q^{-2} Y^{-1} X T_{0}^{2}-q^{-2} Y^{-1} X t_{0} T_{0}-X T_{0} T_{1}+X t_{0} T_{1}, \\
& X^{-1} Y=q^{-2} Y X^{-1}+\left(q-q^{-1}\right) q^{-1} T_{1} T_{3}-q^{-1} T_{0} T_{2}+q^{-1} t_{0} T_{2}-Y^{-1} t_{0} T_{3} \\
& +q^{-2} X T_{0} T_{1}-q^{-2} X t_{0} T_{1}+q^{-2} Y^{-1} T_{0} T_{3}-q^{-2} Y^{-1} X T_{0}^{2}+q^{-2} Y^{-1} X t_{0} T_{0} \text {, } \\
& X^{-1} Y^{-1}=q^{2} Y^{-1} X^{-1}-q^{2} Y^{-1} T_{0} T_{3}+q^{2} Y^{-1} t_{0} T_{3}+q T_{0} T_{2}-q t_{0} T_{2} \\
& -q^{2} X T_{0} T_{1}+q^{2} X t_{0} T_{1}+q^{2} Y^{-1} X T_{0}^{2}-q^{2} Y^{-1} X t_{0} T_{0}, \\
& X Y^{-1}=q^{-2} Y^{-1} X+X T_{0} T_{1}-X t_{0} T_{1}-q^{-2} Y^{-1} X T_{0}^{2}+q^{-2} Y^{-1} X t_{0} T_{0} \\
& +q^{-2} Y^{-1} T_{0} T_{3}-q^{-2} Y^{-1} t_{0} T_{3}-q^{-1} T_{0} T_{2}+q^{-1} t_{0} T_{2} \text {. }
\end{aligned}
$$

Proof. In Proposition 7.1 eliminate $\left\{t_{i}\right\}_{i=1}^{3}$ using the displayed relations 2-4, and eliminate $\left\{C_{i}\right\}_{i \in \mathbb{I}}$ using the displayed relations $13-16$. Simplify the results using the displayed relations $5-8$.

We just gave two versions of a presentation for $\hat{H}_{q}$. From now on we focus on version II. This version will yield our reduction rules and basis for $\hat{H}_{q}$. 
Definition 7.3. The generators $X^{ \pm 1}, Y^{ \pm 1}, t_{0},\left\{T_{i}\right\}_{i \in \mathbb{I}}$ of $\hat{H}_{q}$ are called balanced.

Note 7.4. Referring to the presentation of $\hat{H}_{q}$ from Proposition 7.2, consider the relations which assert that the $\left\{T_{i}\right\}_{i \in \mathbb{I}}$ are central. These relations can be expressed as

$$
\begin{aligned}
& T_{i} X^{ \pm 1}=X^{ \pm 1} T_{i}, \quad T_{i} Y^{ \pm 1}=Y^{ \pm 1} T_{i}, \quad T_{i} t_{0}=t_{0} T_{i}, \quad i \in \mathbb{I}, \\
& T_{i} T_{j}=T_{j} T_{i}, \quad i, j \in \mathbb{I}, \quad i>j .
\end{aligned}
$$

Definition 7.5. By a reduction rule for $\hat{H}_{q}$ we mean an equation that appears in Proposition 7.2 or Note 7.4. Of these reduction rules, the last four in Proposition 7.2 are said to be of the first kind, the preceeding five are said to be of the second kind, and the rest are said to be of the third kind.

Definition 7.6. For an integer $n \geq 0$, by a word of length $n$ in $\hat{H}_{q}$ we mean a product $g_{1} g_{2} \cdots g_{n}$ such that $g_{i}$ is a balanced generator of $\hat{H}_{q}$ for $1 \leq i \leq n$. We interpret the word of length 0 as the multiplicative identity in $\hat{H}_{q}$. A word is called forbidden whenever it is the left-hand side of a reduction rule. Every forbidden word has length two. A forbidden word is said to be of the first kind (resp. second kind) (resp. third kind) whenever the corresponding reduction rule is of the first kind (resp. second kind) (resp. third kind).

Definition 7.7. Let $w$ denote a forbidden word in $\hat{H}_{q}$, and consider the corresponding reduction rule. By a descendent of $w$ we mean a word that appears on the right-hand side of that reduction rule.

Roughly speaking, the following result amounts to a universal analog of [10, Theorem 5.3].

Proposition 7.8. The following is a basis for the $\mathbb{F}$-vector space $\hat{H}_{q}$ :

$$
Y^{i} X^{j} t_{0}^{k} T_{0}^{\ell} T_{1}^{r} T_{2}^{s} T_{3}^{t} \quad i, j \in \mathbb{Z}, \quad k \in\{0,1\}, \quad \ell, r, s, t \in \mathbb{N} .
$$

Proof. We invoke Bergman's Diamond Lemma [3, Theorem 1.2]. Let $g_{1} g_{2} \cdots g_{n}$ denote a word in $\hat{H}_{q}$. This word is called reducible whenever there exists an integer $i(2 \leq i \leq n)$ such that $g_{i-1} g_{i}$ is forbidden. A word is called irreducible whenever it is not reducible. The list (7.2) consists of the irreducible words in $\hat{H}_{q}$. Let $w=g_{1} g_{2} \ldots g_{n}$ denote a word in $\hat{H}_{q}$. By an inversion in $w$ we mean an ordered pair of integers $(i, j)$ such that $1 \leq i<j \leq n$ and the word $g_{i} g_{j}$ is forbidden. The inversion $(i, j)$ is of the first kind (resp. second kind) (resp. third kind) whenever the forbidden word $g_{i} g_{j}$ is of the first kind (resp. second kind) (resp. third kind). Let $W$ denote the set of all words in $\hat{H}_{q}$. We define a partial order $<$ on $W$ as follows. Pick any words $w, w^{\prime}$ in $W$ and write $w=g_{1} g_{2} \cdots g_{n}$. We say that $w$ dominates $w^{\prime}$ whenever there exists an integer $i(2 \leq i \leq n)$ such that $(i-1, i)$ is an inversion for $w$, and $w^{\prime}$ is obtained from $w$ by replacing $g_{i-1} g_{i}$ by one of its descendents. In this case either (i) $w$ has more inversions of the first kind than $w^{\prime}$, or (ii) $w$ and $w^{\prime}$ have the same number of inversions of the first kind, but $w$ has more inversions of the second kind than $w^{\prime}$, or (iii) $w$ and $w^{\prime}$ have the same number of inversions for each of the first and second kind, but $w$ has more inversions of the third kind than $w^{\prime}$. By these comments the transitive closure of the domination relation on $W$ is a partial order on $W$ which we denote by $<$. By construction $<$ is a semigroup partial order [3, p. 181] and satisfies the descending chain condition [3, p. 179]. We now relate the partial order $<$ to our reduction rules. Let $w=g_{1} g_{2} \cdots g_{n}$ denote a reducible word in $\hat{H}_{q}$. Then there exists an integer $i(2 \leq i \leq n)$ such that $g_{i-1} g_{i}$ is forbidden. There exists a reduction rule with $g_{i-1} g_{i}$ on the left-hand side; in $w$ we eliminate $g_{i-1} g_{i}$ using this reduction rule and thereby express $w$ as a linear combination of words, each less than $w$ with respect to $<$. Therefore the reduction rules are compatible with $<$ in the sense of Bergman [3, p. 181]. In order to employ the Diamond Lemma, we must show that the ambiguities are resolvable in the sense of Bergman [3, p. 181]. 
There are potentially two kinds of ambiguities; inclusion ambiguities and overlap ambiguities [3, p. 181]. For the present example there are no inclusion ambiguities. The nontrivial overlap ambiguities are

$$
\begin{array}{lccc}
t_{0} X Y, & t_{0} X^{-1} Y, & t_{0} X Y^{-1}, & t_{0} X^{-1} Y^{-1}, \\
t_{0}^{2} X, & t_{0}^{2} X^{-1}, \quad t_{0}^{2} Y, & t_{0}^{2} Y^{-1}, & \\
X X^{-1} Y, & X X^{-1} Y^{-1}, & X^{-1} X Y, & X^{-1} X Y^{-1}, \\
X Y Y^{-1}, & X Y^{-1} Y, & X^{-1} Y Y^{-1}, & X^{-1} Y^{-1} Y, \\
t_{0} X X^{-1}, & t_{0} X^{-1} X, & t_{0} Y Y^{-1}, & t_{0} Y^{-1} Y .
\end{array}
$$

Take for example $t_{0} X Y$. The words $t_{0} X$ and $X Y$ are forbidden. Therefore $t_{0} X Y$ can be reduced in two ways; we could evaluate $t_{0} X$ first or we could evaluate $X Y$ first. Either way, after a 3 -step reduction we get the same resolution, which is

$$
\begin{array}{rl}
q^{2} Y & X T_{0}+q^{-2} Y X^{-1} T_{0}+q^{2} Y^{-1} X^{-1} t_{0}+q^{2} Y^{-1} X T_{0}-q^{2} X T_{1} \\
& +\left(q^{-2}-1\right) X T_{0}^{2} T_{1}+\left(1-q^{-2}\right) X t_{0} T_{0} T_{1}-X^{-1} T_{1}-Y T_{3}-q^{2} Y^{-1} T_{3} \\
& -\left(q-q^{-1}\right) t_{0} T_{0} T_{2}+\left(1-q^{-2}\right) T_{0} T_{1} T_{3}+q T_{2} .
\end{array}
$$

Therefore the ambiguity $t_{0} X Y$ is resolvable. The other ambiguities listed above are similarly shown to be resolvable. Their resolutions are displayed in the tables below.

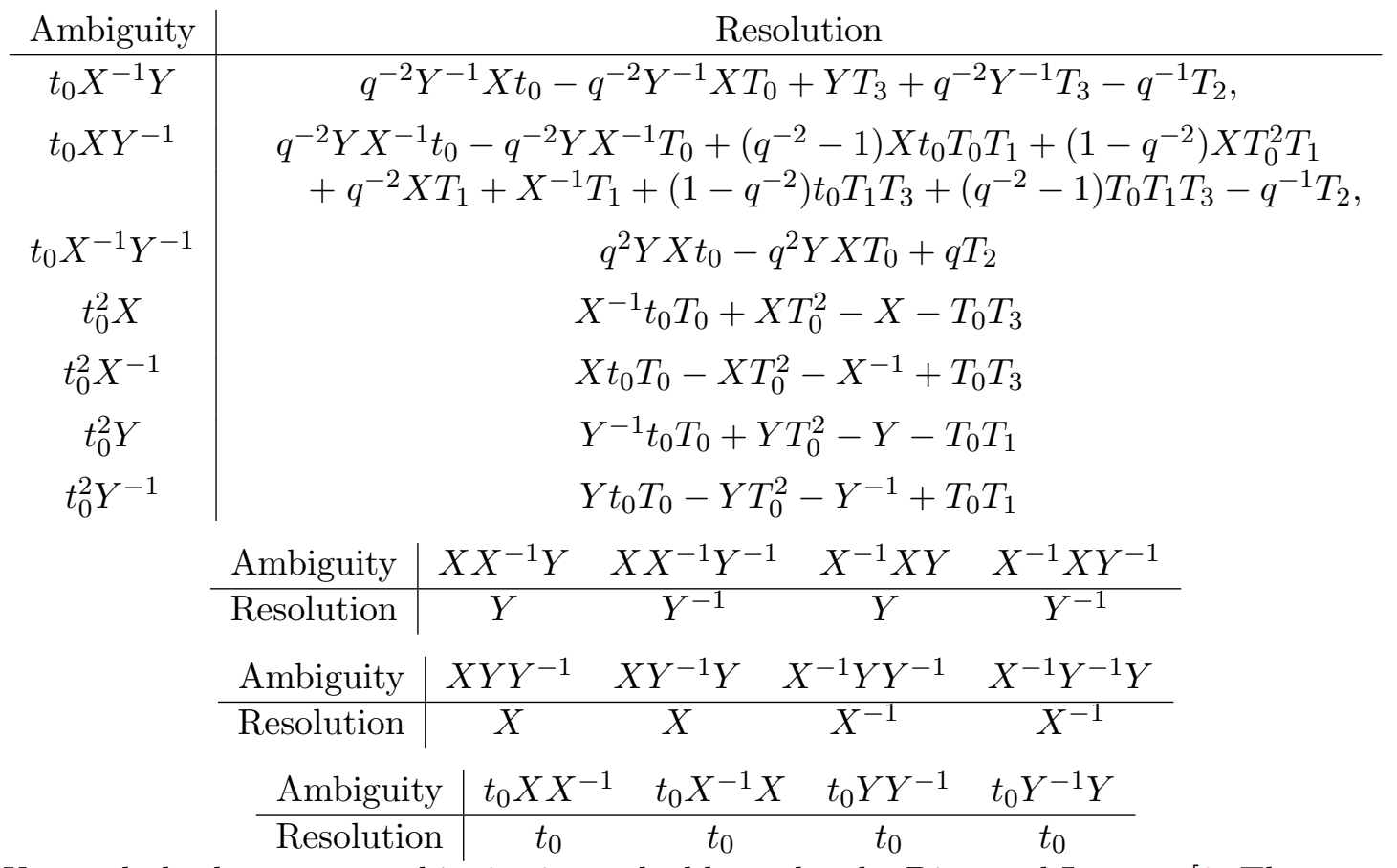

We conclude that every ambiguity is resolvable, so by the Diamond Lemma [3, Theorem 1.2] the irreducible words form a basis for $\hat{H}_{q}$. The result follows.

In Proposition 7.8 we gave a basis for $\hat{H}_{q}$. In Proposition 7.14 below we give a variation on this basis.

Let $\lambda$ denote an indeterminate. Let $\mathbb{F}\left[\lambda, \lambda^{-1}\right]$ denote the $\mathbb{F}$-algebra of Laurent polynomials in $\lambda$ that have all coefficients in $\mathbb{F}$.

Lemma 7.9. The following is a basis for the $\mathbb{F}$-vector space $\mathbb{F}\left[\lambda, \lambda^{-1}\right]$ :

$$
\lambda^{k}\left(\lambda+\lambda^{-1}\right)^{\ell}, \quad k \in\{0,1\}, \quad \ell \in \mathbb{N} .
$$


Proof. The vectors $\left\{\lambda^{i}\right\}_{i \in \mathbb{Z}}$ form a basis for the $\mathbb{F}$-vector space $\mathbb{F}\left[\lambda, \lambda^{-1}\right]$. List the elements of this basis in the following order:

$$
1, \quad \lambda, \quad \lambda^{-1}, \quad \lambda^{2}, \quad \lambda^{-2}, \quad \lambda^{3}, \quad \lambda^{-3}, \quad \ldots
$$

List the elements of (7.3) in the following order:

$$
1, \quad \lambda, \quad \lambda+\lambda^{-1}, \quad \lambda\left(\lambda+\lambda^{-1}\right), \quad\left(\lambda+\lambda^{-1}\right)^{2}, \quad \lambda\left(\lambda+\lambda^{-1}\right)^{2}, \quad \ldots
$$

Write each element of (7.5) as a linear combination of (7.4). Consider the corresponding coefficient matrix. This matrix is upper triangular with all diagonal entries 1 . The result follows.

For a subset $S$ of any algebra let $\langle S\rangle$ denote the subalgebra generated by $S$.

Definition 7.10. Let $\mathbb{T}$ denote the following subalgebra of $\hat{H}_{q}$ :

$$
\mathbb{T}=\left\langle t_{0}^{ \pm 1}, T_{1}, T_{2}, T_{3}\right\rangle .
$$

Let $\left\{\lambda_{i}\right\}_{i=0}^{3}$ denote mutually commuting indeterminates. By construction the $\mathbb{F}$-algebra $\mathbb{T}$ is commutative and generated by $t_{0}^{ \pm 1}, T_{1}, T_{2}, T_{3}$. Therefore there exists a surjective $\mathbb{F}$-algebra homomorphism $\varphi: \mathbb{F}\left[\lambda_{0}^{ \pm 1}, \lambda_{1}, \lambda_{2}, \lambda_{3}\right] \rightarrow \mathbb{T}$ that sends

$$
\lambda_{0}^{ \pm 1} \mapsto t_{0}^{ \pm 1}, \quad \lambda_{1} \mapsto T_{1}, \quad \lambda_{2} \mapsto T_{2}, \quad \lambda_{3} \mapsto T_{3} .
$$

Proposition 7.11. The above homomorphism $\varphi$ is an isomorphism. Moreover, in each line below the displayed vectors form a basis for the $\mathbb{F}$-vector space $\mathbb{T}$ :

$$
\begin{aligned}
& t_{0}^{k} T_{0}^{\ell} T_{1}^{r} T_{2}^{s} T_{3}^{t}, \quad k \in\{0,1\}, \quad \ell, r, s, t \in \mathbb{N} ; \\
& t_{0}^{k} T_{1}^{r} T_{2}^{s} T_{3}^{t}, \quad k \in \mathbb{Z}, \quad r, s, t \in \mathbb{N} .
\end{aligned}
$$

Proof. By Lemma 7.9 the following is a basis for the $\mathbb{F}$-vector space $\mathbb{F}\left[\lambda_{0}^{ \pm 1}, \lambda_{1}, \lambda_{2}, \lambda_{3}\right]$ :

$$
\lambda_{0}^{k}\left(\lambda_{0}+\lambda_{0}^{-1}\right)^{\ell} \lambda_{1}^{r} \lambda_{2}^{s} \lambda_{3}^{t}, \quad k \in\{0,1\}, \quad \ell, r, s, t \in \mathbb{N} .
$$

The homomorphism $\varphi$ sends the vectors (7.8) to the vectors (7.6); therefore the vectors (7.6) span $\mathbb{T}$. The vectors (7.6) are linearly independent by Proposition 7.8. Therefore the vectors (7.6) form a basis for $\mathbb{T}$. Consequently $\varphi$ is an isomorphism and (7.7) is a basis for $\mathbb{T}$.

Recall the elements $\alpha, \beta, \gamma$ of $\hat{H}_{q}$ from (6.6)-(6.8). By those equations $\alpha, \beta, \gamma$ are contained in $\mathbb{T}$. More precisely, (6.6)-(6.8) show how $\alpha, \beta, \gamma$ look in the basis for $\mathbb{T}$ from (7.7). The elements $\alpha, \beta, \gamma$ look as follows in the basis for $\mathbb{T}$ from (7.6):

$$
\begin{aligned}
& \alpha=q T_{0} T_{1}-\left(q-q^{-1}\right) t_{0} T_{1}+T_{2} T_{3}, \quad \beta=q T_{0} T_{3}-\left(q-q^{-1}\right) t_{0} T_{3}+T_{1} T_{2}, \\
& \gamma=q T_{0} T_{2}-\left(q-q^{-1}\right) t_{0} T_{2}+T_{3} T_{1} .
\end{aligned}
$$

We now consider the subalgebras $\left\langle X^{ \pm 1}\right\rangle$ and $\left\langle Y^{ \pm 1}\right\rangle$ of $\hat{H}_{q}$. By Proposition 7.8 the vectors $\left\{X^{i}\right\}_{i \in \mathbb{Z}}$ form a basis for $\left\langle X^{ \pm 1}\right\rangle$ and the vectors $\left\{Y^{i}\right\}_{i \in \mathbb{Z}}$ form a basis for $\left\langle Y^{ \pm 1}\right\rangle$.

Lemma 7.12. There exists an isomorphism of $\mathbb{F}$-algebras $\mathbb{F}\left[\lambda^{ \pm 1}\right] \rightarrow\left\langle X^{ \pm 1}\right\rangle$ that sends $\lambda \mapsto X$. There exists an isomorphism of $\mathbb{F}$-algebras $\mathbb{F}\left[\lambda^{ \pm 1}\right] \rightarrow\left\langle Y^{ \pm 1}\right\rangle$ that sends $\lambda \mapsto Y$.

Proposition 7.13. The $\mathbb{F}$-linear map

$$
\left\langle Y^{ \pm 1}\right\rangle \otimes\left\langle X^{ \pm 1}\right\rangle \otimes \mathbb{T} \rightarrow \hat{H}_{q}, \quad u \otimes v \otimes w \mapsto u v w
$$

is a bijection. 
Proof. By Proposition 7.8, Lemma 7.12, and since (7.6) is a basis for $\mathbb{T}$.

We now give a variation on the basis for $\hat{H}_{q}$ given in Proposition 7.8.

Proposition 7.14. The following is a basis for the $\mathbb{F}$-vector space $\hat{H}_{q}$ :

$$
Y^{i} X^{j} t_{0}^{k} T_{1}^{r} T_{2}^{s} T_{3}^{t}, \quad i, j, k \in \mathbb{Z}, \quad r, s, t \in \mathbb{N} .
$$

Proof. By Proposition 7.13 and since (7.7) is a basis for $\mathbb{T}$.

\section{The coefficient matrix}

Suppose we have an element of $\hat{H}_{q}$ that we wish to express as a linear combination of the vectors (7.2) or (7.9). In order to describe the result efficiently we will use the following notation.

Definition 8.1. By Proposition 7.13 each $h \in \hat{H}_{q}$ can be written as

$$
h=\sum_{i, j \in \mathbb{Z}} Y^{i} X^{j} t_{i j}, \quad t_{i j} \in \mathbb{T} .
$$

Moreover for $i, j \in \mathbb{Z}$ the element $t_{i j}$ is uniquely determined by $h$. We call $t_{i j}$ the coefficient of $Y^{i} X^{j}$ in $h$. The coefficient matrix for $h$ has rows and columns indexed by $\mathbb{Z}$ and $(i, j)$-entry $t_{i j}$

\begin{tabular}{|c|c|c|c|c|c|c|c|}
\hline & $\ldots$ & $X^{-2}$ & $X^{-1}$ & 1 & $X$ & $X^{2}$ & $\ldots$ \\
\hline$\vdots$ & & & & $\vdots$ & & & \\
\hline$Y^{-2}$ & & $t_{-2,-2}$ & $t_{-2,-1}$ & $t_{-2,0}$ & $t_{-2,1}$ & $t_{-2,2}$ & \\
\hline$Y^{-1}$ & & $t_{-1,-2}$ & $t_{-1,-1}$ & $t_{-1,0}$ & $t_{-1,1}$ & $t_{-1,2}$ & \\
\hline 1 & $\ldots$ & $t_{0,-2}$ & $t_{0,-1}$ & $t_{0,0}$ & $t_{0,1}$ & $t_{0,2}$ & . \\
\hline$Y$ & & $t_{1,-2}$ & $t_{1,-1}$ & $t_{1,0}$ & $t_{1,1}$ & $t_{1,2}$ & \\
\hline$Y^{2}$ & & $t_{2,-2}$ & $t_{2,-1}$ & $t_{2,0}$ & $t_{2,1}$ & $t_{2,2}$ & \\
\hline$\vdots$ & & & & $\vdots$ & & & \\
\hline
\end{tabular}
for $i, j \in \mathbb{Z}$. We view

A coefficient matrix has finitely many nonzero entries. When we display a coefficient matrix, any row or column not shown has all entries zero.

Example 8.2. The coefficient matrix for $A$ is

\begin{tabular}{c|ccc} 
& $X^{-1}$ & 1 & $X$ \\
\hline$Y^{-1}$ & 0 & 1 & 0 \\
1 & 0 & 0 & 0 \\
$Y$ & 0 & 1 & 0
\end{tabular}

The coefficient matrix for $B$ is

\begin{tabular}{c|ccc} 
& $X^{-1}$ & 1 & $X$ \\
\hline$Y^{-1}$ & 0 & 0 & 0 \\
1 & 1 & 0 & 1 \\
$Y$ & 0 & 0 & 0
\end{tabular}

Our next goal is to compute the coefficient matrix for $C$. In order to simplify the computation we initially work with an element $\theta \in \hat{H}_{q}$ that is closely related to $C$. 
Definition 8.3. Define $\theta \in \hat{H}_{q}$ such that

$$
q C=\gamma-\theta t_{0}^{-1}
$$

where we recall $\gamma=\left(q^{-1} t_{0}+q t_{0}^{-1}\right) T_{2}+T_{1} T_{3}$.

Lemma 8.4. In the basis (7.9) the element $\theta$ looks as follows:

$$
\theta=Y X^{-1} t_{0}-Y^{-1} X t_{0}^{-1}+Y^{-1} T_{3}+X T_{1}+q^{-1} t_{0}^{2} T_{2} .
$$

Proof. Recall that $C=t_{0} t_{2}+\left(t_{0} t_{2}\right)^{-1}$. We have $t_{0} t_{2}=q^{-1} t_{3}^{-1} T_{1}-q^{-1} Y X^{-1}$ by Lemma 5.11. Also $t_{3}^{-1}=T_{3}-t_{3}$ and $t_{3}=X T_{0}-X t_{0}$. By these comments

$$
t_{0} t_{2}=q^{-1} T_{1} T_{3}-q^{-1} X T_{0} T_{1}+q^{-1} X t_{0} T_{1}-q^{-1} Y X^{-1} .
$$

We have $\left(t_{0} t_{2}\right)^{-1}=q t_{3} T_{1}-q X Y^{-1}$ by Lemma 5.11. We mentioned $t_{3}=X T_{0}-X t_{0}$, and the term $X Y^{-1}$ can be evaluated using a reduction rule from Proposition 7.2. The result follows from these observations along with Definition 8.3.

Lemma 8.5. The coefficient matrix for $\theta$ is

\begin{tabular}{c|ccc} 
& $X^{-1}$ & 1 & $X$ \\
\hline$Y^{-1}$ & 0 & $T_{3}$ & $-t_{0}^{-1}$ \\
1 & 0 & $q^{-1} t_{0}^{2} T_{2}$ & $T_{1}$ \\
$Y$ & $t_{0}$ & 0 & 0
\end{tabular}

Proof. Use Lemma 8.4.

Lemma 8.6. The coefficent matrix for $C$ is

\begin{tabular}{c|ccc} 
& $X^{-1}$ & 1 & $X$ \\
\hline$Y^{-1}$ & 0 & $-q^{-1} t_{0}^{-1} T_{3}$ & $q^{-1} t_{0}^{-2}$ \\
1 & 0 & $t_{0}^{-1} T_{2}+q^{-1} T_{1} T_{3}$ & $-q^{-1} t_{0}^{-1} T_{1}$ \\
$Y$ & $-q^{-1}$ & 0 & 0
\end{tabular}

Proof. Use Definition 8.3 and Lemma 8.5.

Lemma 8.7. The coefficient matrix for $X C$ is

\begin{tabular}{c|ccccc} 
& $X^{-2}$ & $X^{-1}$ & 1 & $X$ & $X^{2}$ \\
\hline$Y^{-2}$ & 0 & 0 & 0 & 0 & 0 \\
$Y^{-1}$ & 0 & $q^{-3} t_{0} T_{3}$ & $-q^{-1} T_{3}^{2}-q^{-3} t_{0}^{2}-q^{-3}$ & $q^{-2}\left(q^{-1} t_{0}+q t_{0}^{-1}\right) T_{3}$ & $-q^{-3}$ \\
1 & 0 & $-q^{-2} t_{0} T_{2}$ & $q^{-1} t_{0} T_{1}+T_{2} T_{3}$ & 0 & 0 \\
$Y$ & 0 & 0 & $-q$ & 0 & 0 \\
$Y^{2}$ & 0 & 0 & 0 & 0 & 0
\end{tabular}

Proof. First find the coefficient matrix for $X \theta$. To do this, in the equation (8.2) multiply each term on the left by $X$ and simplify the result using the reduction rules from Proposition 7.2. This yields the coefficient matrix for $X \theta$. Using this coefficient matrix and (8.1), we routinely obtain the coefficient matrix for $X C$.

We mention two results for later use. 
Lemma 8.8. We have

$$
X^{-1} C=q^{-2} C\left(X+X^{-1}\right)-X C-q^{-1}\left(q^{2}-q^{-2}\right)\left(Y+Y^{-1}\right)+q^{-1}\left(q-q^{-1}\right) \alpha,
$$

where we recall $\alpha=\left(q^{-1} t_{0}+q t_{0}^{-1}\right) T_{1}+T_{2} T_{3}$.

Proof. In the first equation of Lemma 6.6, eliminate $A$ using $A=Y+Y^{-1}$ and $B$ using $B=X+X^{-1}$. In the resulting equation solve for $X^{-1} C$.

Lemma 8.9. Given $h \in \hat{H}_{q}$ and $v \in \mathbb{T}$ such that $h v=0$. Then $h=0$ or $v=0$.

Proof. We assume $v \neq 0$ and show $h=0$. Following Definition 8.1 write

$$
h=\sum_{i, j \in \mathbb{Z}} Y^{i} X^{j} t_{i j}, \quad t_{i j} \in \mathbb{T} .
$$

In this equation we multiply each term on the right by $v$ to obtain

$$
0=\sum_{i, j \in \mathbb{Z}} Y^{i} X^{j} t_{i j} v
$$

Note that $t_{i j} v \in \mathbb{T}$ for $i, j \in \mathbb{Z}$. By this and Proposition 7.13 we find $t_{i j} v=0$ for $i, j \in \mathbb{Z}$. The algebra $\mathbb{T}$ is isomorphic to $\mathbb{F}\left[\lambda_{0}^{ \pm 1}, \lambda_{1}, \lambda_{2}, \lambda_{3}\right]$ by Proposition 7.11 . The algebra $\mathbb{F}\left[\lambda_{0}^{ \pm 1}, \lambda_{1}, \lambda_{2}, \lambda_{3}\right]$ is a domain, so $\mathbb{T}$ is a domain. By this and since $v \neq 0$ we find $t_{i j}=0$ for all $i, j \in \mathbb{Z}$. Therefore $h=0$.

\section{$9 \quad$ The proof of Theorem 4.4}

In this section we prove Theorem 4.4. Recall the Casimir element $\Omega$ in $\Delta_{q}$, from Definition 2.6. Let $\Omega^{\prime}$ denote the element (4.1), so that

$$
\Omega^{\prime}=\left(q+q^{-1}\right)^{2}-\left(q^{-1} t_{0}+q t_{0}^{-1}\right)^{2}-T_{1}^{2}-T_{2}^{2}-T_{3}^{2}-\left(q^{-1} t_{0}+q t_{0}^{-1}\right) T_{1} T_{2} T_{3} .
$$

Theorem 4.4 asserts that $\Omega^{\prime}$ is the image of $\Omega$ under $\psi$.

Proof of Theorem 4.4. By Definition 2.6 and Theorem 4.1 the image of $\Omega$ under $\psi$ is the following element of $\hat{H}_{q}$ :

$$
q^{-1} A C B+q^{-2} A^{2}+q^{-2} B^{2}+q^{2} C^{2}-q^{-1} A \alpha-q^{-1} B \beta-q C \gamma,
$$

where $\alpha, \beta, \gamma$ are from (6.6)-(6.8). We show that (9.1) is equal to $\Omega^{\prime}$. Define $D$ to be (9.1) minus $\Omega^{\prime}$. We show that $D=0$. Our strategy is to find the coefficient matrix for $D$ in the sense of Definition 8.1. Using $A=Y+Y^{-1}$ and $B=X+X^{-1}$ we obtain

$$
\begin{aligned}
D= & q^{-1}\left(Y+Y^{-1}\right) C\left(X+X^{-1}\right)+q^{-2}\left(Y+Y^{-1}\right)^{2}+q^{-2}\left(X+X^{-1}\right)^{2} \\
& +q^{2} C^{2}-q^{-1}\left(Y+Y^{-1}\right) \alpha-q^{-1}\left(X+X^{-1}\right) \beta-q C \gamma-\Omega^{\prime} .
\end{aligned}
$$

In order to evaluate $D$ further we consider the term $C^{2}$. In this product eliminate the first factor using the formula for $C$ from Lemma 8.6. Simplify the result using the fact that $C$ commutes with $t_{0}$; this gives

$$
\begin{aligned}
C^{2}= & q^{-1} Y^{-1} X C t_{0}^{-2}-q^{-1} Y X^{-1} C-q^{-1} X C t_{0}^{-1} T_{1} \\
& -q^{-1} Y^{-1} C t_{0}^{-1} T_{3}+C\left(t_{0}^{-1} T_{2}+q^{-1} T_{1} T_{3}\right) .
\end{aligned}
$$


In the above formula we eliminate $X^{-1} C$ using Lemma 8.8. Evaluating (9.2) using the results we obtain

$$
\begin{aligned}
D= & q C\left(T_{1} T_{3}-\gamma+q t_{0}^{-1} T_{2}\right)-q Y^{-1} C t_{0}^{-1} T_{3}+q^{-1} Y^{-1} C\left(X+X^{-1}\right) \\
& -q X C t_{0}^{-1} T_{1}+q Y X C+q Y^{-1} X C t_{0}^{-2}+G
\end{aligned}
$$

where

$$
\begin{aligned}
G= & q^{2} Y^{2}+q^{-2} Y^{-2}-q Y \alpha-q^{-1} Y^{-1} \alpha+q^{-2} X^{2}+q^{-2} X^{-2} \\
& -q^{-1} X \beta-q^{-1} X^{-1} \beta+q^{2}+3 q^{-2}-\Omega^{\prime}
\end{aligned}
$$

We continue to compute the coefficient matrix of $D$. For the next step we will display the coefficient matrix for a number of elements in $\hat{H}_{q}$. When we display these coefficient matrices we just display the $(i, j)$ entry for $-2 \leq i, j \leq 2$, since it turns out that all the other entries are zero. Consider the element $C$ of $\hat{H}_{q}$. By Lemma 8.6 the coefficient matrix for $C$ is

\begin{tabular}{c|ccccc} 
& $X^{-2}$ & $X^{-1}$ & 1 & $X$ & $X^{2}$ \\
\hline$Y^{-2}$ & 0 & 0 & 0 & 0 & 0 \\
$Y^{-1}$ & 0 & 0 & $-q^{-1} t_{0}^{-1} T_{3}$ & $q^{-1} t_{0}^{-2}$ & 0 \\
1 & 0 & 0 & $t_{0}^{-1} T_{2}+q^{-1} T_{1} T_{3}$ & $-q^{-1} t_{0}^{-1} T_{1}$ & 0 \\
$Y$ & 0 & $-q^{-1}$ & 0 & 0 & 0 \\
$Y^{2}$ & 0 & 0 & 0 & 0 & 0
\end{tabular}

The coefficient matrix for $Y^{-1} C$ is

\begin{tabular}{c|ccccc} 
& $X^{-2}$ & $X^{-1}$ & 1 & $X$ & $X^{2}$ \\
\hline$Y^{-2}$ & 0 & 0 & $-q^{-1} t_{0}^{-1} T_{3}$ & $q^{-1} t_{0}^{-2}$ & 0 \\
$Y^{-1}$ & 0 & 0 & $t_{0}^{-1} T_{2}+q^{-1} T_{1} T_{3}$ & $-q^{-1} t_{0}^{-1} T_{1}$ & 0 \\
1 & 0 & $-q^{-1}$ & 0 & 0 & 0 \\
$Y$ & 0 & 0 & 0 & 0 & 0 \\
$Y^{2}$ & 0 & 0 & 0 & 0 & 0
\end{tabular}

By this and since $t_{0}$ commutes with $X+X^{-1}$, the coefficient matrix for $Y^{-1} C\left(X+X^{-1}\right)$ is

\begin{tabular}{c|ccccc} 
& $X^{-2}$ & $X^{-1}$ & 1 & $X$ & $X^{2}$ \\
\hline$Y^{-2}$ & 0 & $-q^{-1} t_{0}^{-1} T_{3}$ & $q^{-1} t_{0}^{-2}$ & $-q^{-1} t_{0}^{-1} T_{3}$ & $q^{-1} t_{0}^{-2}$ \\
$Y^{-1}$ & 0 & $t_{0}^{-1} T_{2}+q^{-1} T_{1} T_{3}$ & $-q^{-1} t_{0}^{-1} T_{1}$ & $t_{0}^{-1} T_{2}+q^{-1} T_{1} T_{3}$ & $-q^{-1} t_{0}^{-1} T_{1}$ \\
1 & $-q^{-1}$ & 0 & $-q^{-1}$ & 0 & 0 \\
$Y$ & 0 & 0 & 0 & 0 & 0 \\
$Y^{2}$ & 0 & 0 & 0 & 0 & 0
\end{tabular}

By Lemma 8.7 the coefficient matrix for $X C$ is

\begin{tabular}{c|ccccc} 
& $X^{-2}$ & $X^{-1}$ & 1 & $X$ & $X^{2}$ \\
\hline$Y^{-2}$ & 0 & 0 & 0 & 0 & 0 \\
$Y^{-1}$ & 0 & $q^{-3} t_{0} T_{3}$ & $-q^{-1} T_{3}^{2}-q^{-3} t_{0}^{2}-q^{-3}$ & $q^{-2}\left(q^{-1} t_{0}+q t_{0}^{-1}\right) T_{3}$ & $-q^{-3}$ \\
1 & 0 & $-q^{-2} t_{0} T_{2}$ & $q^{-1} t_{0} T_{1}+T_{2} T_{3}$ & 0 & 0 \\
$Y$ & 0 & 0 & $-q$ & 0 & 0 \\
$Y^{2}$ & 0 & 0 & 0 & 0 & 0
\end{tabular}


The coefficient matrix for $Y X C$ is

\begin{tabular}{c|ccccc} 
& $X^{-2}$ & $X^{-1}$ & 1 & $X$ & $X^{2}$ \\
\hline$Y^{-2}$ & 0 & 0 & 0 & 0 & 0 \\
$Y^{-1}$ & 0 & 0 & 0 & 0 & 0 \\
1 & 0 & $q^{-3} t_{0} T_{3}$ & $-q^{-1} T_{3}^{2}-q^{-3} t_{0}^{2}-q^{-3}$ & $q^{-2}\left(q^{-1} t_{0}+q t_{0}^{-1}\right) T_{3}$ & $-q^{-3}$ \\
$Y$ & 0 & $-q^{-2} t_{0} T_{2}$ & $q^{-1} t_{0} T_{1}+T_{2} T_{3}$ & 0 & 0 \\
$Y^{2}$ & 0 & 0 & $-q$ & 0 & 0
\end{tabular}

The coefficient matrix for $Y^{-1} X C$ is

\begin{tabular}{c|ccccc} 
& $X^{-2}$ & $X^{-1}$ & 1 & $X$ & $X^{2}$ \\
\hline$Y^{-2}$ & 0 & $q^{-3} t_{0} T_{3}$ & $-q^{-1} T_{3}^{2}-q^{-3} t_{0}^{2}-q^{-3}$ & $q^{-2}\left(q^{-1} t_{0}+q t_{0}^{-1}\right) T_{3}$ & $-q^{-3}$ \\
$Y^{-1}$ & 0 & $-q^{-2} t_{0} T_{2}$ & $q^{-1} t_{0} T_{1}+T_{2} T_{3}$ & 0 & 0 \\
1 & 0 & 0 & $-q$ & 0 & 0 \\
$Y$ & 0 & 0 & 0 & 0 & 0 \\
$Y^{2}$ & 0 & 0 & 0 & 0 & 0
\end{tabular}

By (9.4) the coefficient matrix for $G$ is

\begin{tabular}{c|ccccc} 
& $X^{-2}$ & $X^{-1}$ & 1 & $X$ & $X^{2}$ \\
\hline$Y^{-2}$ & 0 & 0 & $q^{-2}$ & 0 & 0 \\
$Y^{-1}$ & 0 & 0 & $-q^{-1} \alpha$ & 0 & 0 \\
1 & $q^{-2}$ & $-q^{-1} \beta$ & $q^{2}+3 q^{-2}-\Omega^{\prime}$ & $-q^{-1} \beta$ & $q^{-2}$ \\
$Y$ & 0 & 0 & $-q \alpha$ & 0 & 0 \\
$Y^{2}$ & 0 & 0 & $q^{2}$ & 0 & 0
\end{tabular}

We now evaluate (9.3) using (9.5)-(9.11). One routinely checks that (9.5) times $q\left(T_{1} T_{3}-\gamma+\right.$ $q t_{0}^{-1} T_{2}$ ) minus (9.6) times $q t_{0}^{-1} T_{3}$ plus (9.7) times $q^{-1}$ minus (9.8) times $q t_{0}^{-1} T_{1}$ plus (9.9) times $q$ plus (9.10) times $q t_{0}^{-2}$ plus (9.11) is equal to zero. Evaluating (9.3) in this light we find that the coefficient matrix of $D$ is zero. Therefore $D=0$ and the result follows.

From now on we retain the notation $\Omega$ for its image under the map $\psi: \Delta_{q} \rightarrow \hat{H}_{q}$. Thus the element $\Omega$ of $\hat{H}_{q}$ satisfies

$$
\Omega=\left(q+q^{-1}\right)^{2}-\left(q^{-1} t_{0}+q t_{0}^{-1}\right)^{2}-T_{1}^{2}-T_{2}^{2}-T_{3}^{2}-\left(q^{-1} t_{0}+q t_{0}^{-1}\right) T_{1} T_{2} T_{3} .
$$

\section{Some results concerning algebraic independence}

Our next general goal is to prove Theorem 4.5. The proof will be completed in Section 12. In the present section we establish some results about algebraic independence that will be used in the proof.

Let $\left\{x_{i}\right\}_{i=1}^{4}$ denote mutually commuting indeterminates. Motivated by the form of (6.6)-(6.8) and (9.12) we consider the following elements in $\mathbb{F}\left[x_{1}, x_{2}, x_{3}, x_{4}\right]$ :

$$
\begin{aligned}
& y_{1}=x_{1} x_{2} x_{3} x_{4}+x_{1}^{2}+x_{2}^{2}+x_{3}^{2}+x_{4}^{2}, \\
& y_{2}=x_{1} x_{2}+x_{3} x_{4}, \quad y_{3}=x_{1} x_{3}+x_{2} x_{4}, \quad y_{4}=x_{1} x_{4}+x_{2} x_{3} .
\end{aligned}
$$

Lemma 10.1 ([16, Lemma 8.1]). The elements $\left\{y_{i}\right\}_{i=1}^{4}$ in (10.1), (10.2) are algebraically independent over $\mathbb{F}$.

Recall the algebra $\mathbb{T}$ from Definition 7.10. 
Lemma 10.2. The following are algebraically independent elements of $\mathbb{T}$ :

$\Omega, \quad \alpha, \quad \beta, \quad \gamma$.

Proof. Recall that $\mathbb{T}$ is generated by $t_{0}^{ \pm 1}, T_{1}, T_{2}, T_{3}$. By Proposition 7.11 the following are algebraically independent over $\mathbb{F}$ :

$t_{0}, \quad T_{1}, \quad T_{2}, \quad T_{3}$

Therefore the following are algebraically independent over $\mathbb{F}$ :

$$
q^{-1} t_{0}+q t_{0}^{-1}, \quad T_{1}, \quad T_{2}, \quad T_{3} .
$$

Denote the sequence (10.3) by $\left\{X_{i}\right\}_{i=1}^{4}$. By Lemma 10.1 the following are algebraically independent over $\mathbb{F}$ :

$$
\begin{aligned}
& X_{1} X_{2} X_{3} X_{4}+X_{1}^{2}+X_{2}^{2}+X_{3}^{2}+X_{4}^{2}, \quad X_{1} X_{2}+X_{3} X_{4}, \\
& X_{1} X_{3}+X_{2} X_{4}, \quad X_{1} X_{4}+X_{2} X_{3} .
\end{aligned}
$$

By (6.6)-(6.8) and (9.12) the above four elements are

$$
\left(q+q^{-1}\right)^{2}-\Omega, \quad \alpha, \quad \beta, \quad \gamma .
$$

The result follows.

Definition 10.3. Let $\mathbb{P}$ denote the following subalgebra of $\mathbb{T}$ :

$$
\mathbb{P}=\langle\Omega, \alpha, \beta, \gamma\rangle
$$

We set some notation. For subspaces $U, V$ of $\hat{H}_{q}$ define $U V=\operatorname{Span}_{\mathbb{F}}\{u v \mid u \in U, v \in V\}$.

In order to motivate the next few sections let us briefly return to the map $\psi: \Delta_{q} \rightarrow \hat{H}_{q}$ from Theorem 4.1. Our current goal is to show that $\psi$ is injective. Recall that $\Delta_{q}$ is generated by $A$, $B, C$. Therefore the image of $\Delta_{q}$ under $\psi$ is the subalgebra $\langle A, B, C\rangle$ of $\hat{H}_{q}$. By Theorem 2.20 the vectors (2.6) form a basis for $\Delta_{q}$. Applying $\psi$ to this basis, we find that the following vectors $\operatorname{span}\langle A, B, C\rangle$ :

$$
A^{i} C^{j} B^{k} \Omega^{\ell} \alpha^{r} \beta^{s} \gamma^{t}, \quad j \in\{0,1\}, \quad i, k, \ell, r, s, t \in \mathbb{N} .
$$

Consequently

$$
\langle A, B, C\rangle=\langle A\rangle\langle B\rangle \mathbb{P}+\langle A\rangle C\langle B\rangle \mathbb{P} .
$$

In order to show that $\psi$ is injective, it suffices to show that the vectors (10.4) are linearly independent. To show this, it will be convenient to expand our focus from the algebra $\langle A, B, C\rangle$ to the algebra $\langle A, B, C, \mathbb{T}\rangle=\left\langle A, B, C, t_{0}^{ \pm 1}, T_{1}, T_{2}, T_{3}\right\rangle$. By (10.5), and since everything in $\langle A, B, C\rangle$ commutes with everything in $\mathbb{T}$,

$$
\langle A, B, C, \mathbb{T}\rangle=\langle A\rangle\langle B\rangle \mathbb{T}+\langle A\rangle C\langle B\rangle \mathbb{T} .
$$

We will show that the following is a basis for the $\mathbb{F}$-vector space $\langle A, B, C, \mathbb{T}\rangle$ :

$$
A^{i} C^{j} B^{k} t_{0}^{\ell} T_{1}^{r} T_{2}^{s} T_{3}^{t}, \quad j \in\{0,1\}, \quad \ell \in \mathbb{Z}, \quad i, k, r, s, t \in \mathbb{N} .
$$

It will follow from this and Lemma 10.2 that the vectors (10.4) are linearly independent. 


\section{The structure of $\hat{H}_{q}$}

In this section we establish some results about $\hat{H}_{q}$ that will be used in the proof of Theorem 4.5. Recall $A=Y+Y^{-1}$ and $B=X+X^{-1}$.

Lemma 11.1. The following is a basis for $\left\langle Y^{ \pm 1}\right\rangle$ :

$$
Y^{k} A^{\ell}, \quad k \in\{0,1\}, \quad \ell \in \mathbb{N} \text {. }
$$

The following is a basis for $\left\langle X^{ \pm 1}\right\rangle$ :

$$
X^{k} B^{\ell}, \quad k \in\{0,1\}, \quad \ell \in \mathbb{N} .
$$

Proof. Combine Lemma 7.9 and Lemma 7.12.

Lemma 11.2. The following sums are direct:

$$
\left\langle Y^{ \pm 1}\right\rangle=\langle A\rangle+Y\langle A\rangle, \quad\left\langle X^{ \pm 1}\right\rangle=\langle B\rangle+X\langle B\rangle .
$$

For each summand a basis is given in the table below.

\begin{tabular}{c|cc} 
subspace & \multicolumn{2}{|c}{ basis } \\
\hline$\langle A\rangle$ & $A^{i}$ & $i \in \mathbb{N}$ \\
$Y\langle A\rangle$ & $Y A^{i}$ & $i \in \mathbb{N}$ \\
$\langle B\rangle$ & $B^{i}$ & $i \in \mathbb{N}$ \\
$X\langle B\rangle$ & $X B^{i}$ & $i \in \mathbb{N}$
\end{tabular}

Proof. Use Lemma 11.1.

Proposition 11.3. The following sum is direct:

$$
\hat{H}_{q}=\langle A\rangle\langle B\rangle \mathbb{T}+\langle A\rangle X\langle B\rangle \mathbb{T}+\langle A\rangle Y\langle B\rangle \mathbb{T}+\langle A\rangle Y X\langle B\rangle \mathbb{T} .
$$

For each summand a basis is given in the table below.

\begin{tabular}{c|ccc} 
subspace & \multicolumn{3}{|c}{ basis } \\
\hline$\langle A\rangle\langle B\rangle \mathbb{T}$ & $A^{i} B^{j} t_{0}^{k} T_{1}^{r} T_{2}^{s} T_{3}^{t}$ & $k \in \mathbb{Z}$, & $i, j, r, s, t \in \mathbb{N}$ \\
$\langle A\rangle X\langle B\rangle \mathbb{T}$ & $A^{i} X B^{j} t_{0}^{k} T_{1}^{r} T_{2}^{s} T_{3}^{t}$ & $k \in \mathbb{Z}$, & $i, j, r, s, t \in \mathbb{N}$ \\
$\langle A\rangle Y\langle B\rangle \mathbb{T}$ & $A^{i} Y B^{j} t_{0}^{k} T_{1}^{r} T_{2}^{s} T_{3}^{t}$ & $k \in \mathbb{Z}$, & $i, j, r, s, t \in \mathbb{N}$ \\
$\langle A\rangle Y X\langle B\rangle \mathbb{T}$ & $A^{i} Y X B^{j} t_{0}^{k} T_{1}^{r} T_{2}^{s} T_{3}^{t}$ & $k \in \mathbb{Z}$, & $i, j, r, s, t \in \mathbb{N}$
\end{tabular}

Proof. By Proposition 7.13, Lemma 11.2, and since (7.7) is a basis for $\mathbb{T}$.

Proposition 11.4. For $\nu \in\{1, X, Y, Y X\}$ the $\mathbb{F}$-linear map

$$
\langle A\rangle \otimes\langle B\rangle \otimes \mathbb{T} \rightarrow\langle A\rangle \nu\langle B\rangle \mathbb{T}, \quad u \otimes v \otimes w \mapsto u \nu v w
$$

is a bijection.

Proof. Use the bases displayed in the table of Proposition 11.3.

Consider the four summands in the decomposition (11.1). For each summand we now consider the corresponding projection map. 
Definition 11.5. For $\nu \in\{1, X, Y, Y X\}$ define an $\mathbb{F}$-linear map $\pi_{\nu}: \hat{H}_{q} \rightarrow \hat{H}_{q}$ such that $\pi_{\nu}$ acts as the identity on $\langle A\rangle \nu\langle B\rangle \mathbb{T}$, and as 0 on the other three summands in (11.1). Thus $\pi_{\nu}$ is the projection from $\hat{H}_{q}$ onto $\langle A\rangle \nu\langle B\rangle \mathbb{T}$. For $h \in \hat{H}_{q}$ we have

$$
\pi_{\nu}(A h)=A \pi_{\nu}(h), \quad \pi_{\nu}(h B)=\pi_{\nu}(h) B, \quad \pi_{\nu}(h v)=\pi_{\nu}(h) v, \quad \forall v \in \mathbb{T} .
$$

Moreover

$$
h=\pi_{1}(h)+\pi_{X}(h)+\pi_{Y}(h)+\pi_{Y X}(h) .
$$

Lemma 11.6. For $\nu \in\{1, X, Y, Y X\}$ the projections $\pi_{\nu}(A), \pi_{\nu}(B), \pi_{\nu}(C)$ are given in the table below.

\begin{tabular}{c|ccc}
$\nu$ & $\pi_{\nu}(A)$ & $\pi_{\nu}(B)$ & $\pi_{\nu}(C)$ \\
\hline 1 & $A$ & $B$ & $q^{-1} \gamma-q^{-2} t_{0} T_{2}-q^{-1} A t_{0}^{-1} T_{3}$ \\
$X$ & 0 & 0 & $q^{-1} A X t_{0}^{-2}-q^{-1} X t_{0}^{-1} T_{1}$ \\
$Y$ & 0 & 0 & $q^{-1} Y t_{0}^{-1} T_{3}-q^{-1} Y B$ \\
$Y X$ & 0 & 0 & $q^{-1} Y X\left(1-t_{0}^{-2}\right)$
\end{tabular}

Proof. To get $\pi_{\nu}(A)$ and $\pi_{\nu}(B)$, note that each of $A, B$ is contained in $\langle A\rangle\langle B\rangle \mathbb{T}$. To get $\pi_{\nu}(C)$, consider the formula for $C$ from Lemma 8.6. In this formula eliminate $X^{-1}, Y^{-1}$ using $X^{-1}=$ $B-X$ and $Y^{-1}=A-Y$.

\section{The proof of Theorem 4.5}

In this section we will prove Theorem 4.5. To prepare for the proof, consider the following subspace of $\hat{H}_{q}$ :

$$
\tilde{H}_{q}=\langle A\rangle\langle B\rangle \mathbb{T}+\langle A\rangle X\langle B\rangle \mathbb{T}+\langle A\rangle Y\langle B\rangle \mathbb{T}+\langle A\rangle Y X\langle B\rangle \mathbb{T}\left(1-t_{0}^{-2}\right) .
$$

Lemma 12.1. The sum in (12.1) is direct.

Proof. Observe that $\mathbb{T}\left(1-t_{0}^{-2}\right)$ is contained in $\mathbb{T}$, so $\langle A\rangle Y X\langle B\rangle \mathbb{T}\left(1-t_{0}^{-2}\right)$ is contained in $\langle A\rangle Y X\langle B\rangle \mathbb{T}$. The result follows in view of Proposition 11.3.

Note that $\mathbb{F}\left[\lambda^{ \pm 1}\right]\left(1-\lambda^{-2}\right)$ is an ideal in $\mathbb{F}\left[\lambda^{ \pm 1}\right]$.

Lemma 12.2. The following sum is direct:

$$
\mathbb{F}\left[\lambda^{ \pm 1}\right]=\mathbb{F} 1+\mathbb{F} \lambda^{-1}+\mathbb{F}\left[\lambda^{ \pm 1}\right]\left(1-\lambda^{-2}\right) .
$$

In other words, the vectors $1, \lambda^{-1}$ form a basis for a complement of $\mathbb{F}\left[\lambda^{ \pm 1}\right]\left(1-\lambda^{-2}\right)$ in $\mathbb{F}\left[\lambda^{ \pm 1}\right]$.

Proof. One checks that the vectors

$$
1, \quad \lambda^{-1}, \quad 1-\lambda^{-2}, \quad \lambda\left(1-\lambda^{-2}\right), \quad \lambda^{-1}\left(1-\lambda^{-2}\right), \quad \lambda^{2}\left(1-\lambda^{-2}\right), \quad \lambda^{-2}\left(1-\lambda^{-2}\right), \quad \ldots
$$

form a basis for $\mathbb{F}\left[\lambda^{ \pm 1}\right]$.

Note that $\mathbb{T}\left(1-t_{0}^{-2}\right)$ is an ideal in $\mathbb{T}$.

Lemma 12.3. The following is a basis for the $\mathbb{F}$-vector space $\mathbb{T}\left(1-t_{0}^{-2}\right)$ :

$$
t_{0}^{k}\left(1-t_{0}^{-2}\right) T_{1}^{r} T_{2}^{s} T_{3}^{t}, \quad k \in \mathbb{Z}, \quad r, s, t \in \mathbb{N} .
$$

The following is a basis for a complement of $\mathbb{T}\left(1-t_{0}^{-2}\right)$ in $\mathbb{T}$ :

$$
t_{0}^{-k} T_{1}^{r} T_{2}^{s} T_{3}^{t}, \quad k \in\{0,1\}, \quad r, s, t \in \mathbb{N} .
$$


Proof. By Lemma 12.2 and the first assertion of Proposition 7.11.

Proposition 12.4. The following is a basis for the $\mathbb{F}$-vector space $\langle A\rangle Y X\langle B\rangle \mathbb{T}\left(1-t_{0}^{-2}\right)$ :

$$
A^{i} Y X B^{j} t_{0}^{k}\left(1-t_{0}^{-2}\right) T_{1}^{r} T_{2}^{s} T_{3}^{t}, \quad k \in \mathbb{Z}, \quad i, j, r, s, t \in \mathbb{N} .
$$

The following is a basis for a complement of $\langle A\rangle Y X\langle B\rangle \mathbb{T}\left(1-t_{0}^{-2}\right)$ in $\langle A\rangle Y X\langle B\rangle \mathbb{T}$ :

$$
A^{i} Y X B^{j} t_{0}^{-k} T_{1}^{r} T_{2}^{s} T_{3}^{t}, \quad k \in\{0,1\}, \quad i, j, r, s, t \in \mathbb{N} .
$$

Proof. Use Proposition 11.4 with $\nu=Y X$. Evaluate this using Lemma 12.3 along with the fact that $\left\{A^{i}\right\}_{i \in \mathbb{N}}$ is a basis for $\langle A\rangle$ and $\left\{B^{i}\right\}_{i \in \mathbb{N}}$ is a basis for $\langle B\rangle$.

Corollary 12.5. The following is a basis for a complement of $\tilde{H}_{q}$ in $\hat{H}_{q}$ :

$$
A^{i} Y X B^{j} t_{0}^{-k} T_{1}^{r} T_{2}^{s} T_{3}^{t}, \quad k \in\{0,1\}, \quad i, j, r, s, t \in \mathbb{N} .
$$

Proof. This follows from the first assertion of Proposition 11.3, the definition of $\tilde{H}_{q}$ in equation (12.1), and the last assertion of Proposition 12.4.

Lemma 12.6. The following $(i)-(i v)$ hold:

(i) $C \in \tilde{H}_{q}$.

(ii) $A \tilde{H}_{q} \subseteq \tilde{H}_{q}$.

(iii) $\tilde{H}_{q} B \subseteq \tilde{H}_{q}$.

(iv) $\tilde{H}_{q} \mathbb{T} \subseteq \tilde{H}_{q}$.

Proof. (i) From the column on the right in the table of Lemma 11.6.

(ii), (iv) By equation (12.1).

(iii) By equation (12.1), and since $B$ commutes with everything in $\mathbb{T}$.

We are about to define an $\mathbb{F}$-linear map $\phi: \tilde{H}_{q} \rightarrow \tilde{H}_{q}$. To define $\phi$ we give its action on the four summands in (12.1). As we will see, the map $\phi$ acts on the first three summands as a scalar multiple of the identity. To give the action of $\phi$ on the fourth summand, we specify what $\phi$ does to the basis for this space given in (12.2).

Definition 12.7. We define an $\mathbb{F}$-linear map $\phi: \tilde{H}_{q} \rightarrow \tilde{H}_{q}$ such that both

(i) $\phi$ acts as $-q^{-1}$ times the identity on

$$
\langle A\rangle\langle B\rangle \mathbb{T}+\langle A\rangle X\langle B\rangle \mathbb{T}+\langle A\rangle Y\langle B\rangle \mathbb{T}
$$

(ii) for $k \in \mathbb{Z}$ and $i, j, r, s, t \in \mathbb{N}$ the map $\phi$ sends

$$
A^{i} Y X B^{j} t_{0}^{k}\left(1-t_{0}^{-2}\right) T_{1}^{r} T_{2}^{s} T_{3}^{t} \mapsto A^{i} C B^{j} t_{0}^{k} T_{1}^{r} T_{2}^{s} T_{3}^{t} .
$$

Note 12.8. The map $\phi$ is characterized as follows. Observe that $\phi: \tilde{H}_{q} \rightarrow \tilde{H}_{q}$ is the unique $\mathbb{F}$-linear map that sends

$$
1 \mapsto-q^{-1}, \quad X \mapsto-q^{-1} X, \quad Y \mapsto-q^{-1} Y, \quad Y X\left(1-t_{0}^{-2}\right) \mapsto C
$$

and satisfies the following for all $h \in \tilde{H}_{q}$ :

$$
\phi(A h)=A \phi(h), \quad \phi(h B)=\phi(h) B, \quad \phi(h u)=\phi(h) u, \quad \forall u \in \mathbb{T} .
$$


Lemma 12.9. We have $\phi^{2}=q^{-2} 1$. Moreover $\phi$ is a bijection.

Proof. The first assertion is routinely checked using the column on the right in the table of Lemma 11.6, along with Definition 12.7. The second assertion is immediate from the first.

Lemma 12.10. Referring to the sum in (12.1), for each summand $U$ the image of $U$ under $\phi$ is displayed in the table below.

\begin{tabular}{c|c}
$U$ & image of $U$ under $\phi$ \\
\hline$\langle A\rangle\langle B\rangle \mathbb{T}$ & $\langle A\rangle\langle B\rangle \mathbb{T}$ \\
$\langle A\rangle X\langle B\rangle \mathbb{T}$ & $\langle A\rangle X\langle B\rangle \mathbb{T}$ \\
$\langle A\rangle Y\langle B\rangle \mathbb{T}$ & $\langle A\rangle Y\langle B\rangle \mathbb{T}$ \\
$\langle A\rangle Y X\langle B\rangle \mathbb{T}\left(1-t_{0}^{-2}\right)$ & $\langle A\rangle C\langle B\rangle \mathbb{T}$
\end{tabular}

Proof. Use Definition 12.7.

Proposition 12.11. The following sum is direct:

$$
\tilde{H}_{q}=\langle A\rangle\langle B\rangle \mathbb{T}+\langle A\rangle X\langle B\rangle \mathbb{T}+\langle A\rangle Y\langle B\rangle \mathbb{T}+\langle A\rangle C\langle B\rangle \mathbb{T} .
$$

Moreover the following is a basis for the $\mathbb{F}$-vector space $\langle A\rangle C\langle B\rangle \mathbb{T}$ :

$$
A^{i} C B^{j} t_{0}^{k} T_{1}^{r} T_{2}^{s} T_{3}^{t}, \quad k \in \mathbb{Z}, \quad i, j, r, s, t \in \mathbb{N} .
$$

Proof. The first assertion is a consequence of Lemma 12.1 and Lemma 12.10, together with the fact that $\phi$ is a bijection. The second assertion follows from Definition 12.7( $i i)$ and the fact that $\phi$ is a bijection.

Proposition 12.12. The sum (10.6) is direct.

Proof. The two summands in (10.6) are included among the four summands in the direct sum (12.3).

Roughly speaking, the following result amounts to a universal analog of [11, Theorem 2.6].

Proposition 12.13. The following is a basis for the $\mathbb{F}$-vector space $\langle A, B, C, \mathbb{T}\rangle$ :

$$
A^{i} C^{j} B^{k} t_{0}^{\ell} T_{1}^{r} T_{2}^{s} T_{3}^{t}, \quad j \in\{0,1\}, \quad \ell \in \mathbb{Z}, \quad i, k, r, s, t \in \mathbb{N} .
$$

Proof. The set of vectors (12.5) consists of the basis for $\langle A\rangle\langle B\rangle \mathbb{T}$ from the table of Proposition 11.3, together with the basis for $\langle A\rangle C\langle B\rangle \mathbb{T}$ from (12.4). The result follows in view of Proposition 12.12.

Proof of Theorem 4.5. By Theorem 2.20 the vectors (2.6) form a basis for $\Delta_{q}$. Applying $\psi$ to this basis, we obtain the following vectors in $\hat{H}_{q}$ :

$$
A^{i} C^{j} B^{k} \Omega^{\ell} \alpha^{r} \beta^{s} \gamma^{t}, \quad j \in\{0,1\}, \quad i, k, \ell, r, s, t \in \mathbb{N} .
$$

These vectors are linearly independent by Lemma 10.2 and since the vectors (12.5) are linearly independent. Therefore $\psi$ is injective. 


\section{The elements in $\hat{H}_{q}$ that commute with $t_{0}$}

We have now proven the five theorems from Section 4. Recall that these theorems describe the map $\psi: \Delta_{q} \rightarrow \hat{H}_{q}$. Our goal for the remainder of the paper is to obtain three extra results about $\hat{H}_{q}$; these results help to illuminate $\psi$ and may be of independent interest. The first extra result concerns the subalgebra $\langle A, B, C, \mathbb{T}\rangle$ of $\hat{H}_{q}$. This subalgebra was first mentioned at the end of Section 10, and a basis for it was given in Proposition 12.13. Our goal for the present section is to show that

$$
\langle A, B, C, \mathbb{T}\rangle=\left\{h \in \hat{H}_{q} \mid t_{0} h=h t_{0}\right\}
$$

We will be discussing the $\mathbb{F}$-linear map $\hat{H}_{q} \rightarrow \hat{H}_{q}, h \mapsto t_{0} h-h t_{0}^{-1}$.

Lemma 13.1. For $\nu \in\{1, X, Y, Y X\}$ the element $t_{0} \nu-\nu t_{0}^{-1}$ is given in the table below.

\begin{tabular}{c|c}
$\nu$ & $t_{0} \nu-\nu t_{0}^{-1}$ \\
\hline 1 & $t_{0}-t_{0}^{-1}$ \\
$X$ & $B t_{0}-T_{3}$ \\
$Y$ & $A t_{0}-T_{1}$ \\
$Y X$ & $q\left(C t_{0}-T_{2}\right)+\left(A B-T_{1} T_{3}\right) t_{0}$
\end{tabular}

Moreover

$$
\left(A B-T_{1} T_{3}\right) t_{0}=A\left(B t_{0}-T_{3}\right)+\left(A t_{0}-T_{1}\right) t_{0} T_{3}-A t_{0}\left(t_{0}-t_{0}^{-1}\right) T_{3}
$$

Proof. The table is obtained using Lemma 5.10. Equation (13.1) is routinely checked.

Lemma 13.2. Under the map $h \mapsto t_{0} h-h t_{0}^{-1}$ the image of $\hat{H}_{q}$ is

$$
\langle A\rangle\left(t_{0}-t_{0}^{-1}\right)\langle B\rangle \mathbb{T}+\langle A\rangle\left(A-t_{0}^{-1} T_{1}\right)\langle B\rangle \mathbb{T}+\langle A\rangle\left(B-t_{0}^{-1} T_{3}\right)\langle B\rangle \mathbb{T}+\langle A\rangle\left(C-t_{0}^{-1} T_{2}\right)\langle B\rangle \mathbb{T} .
$$

This image is contained in $\langle A, B, C, \mathbb{T}\rangle$.

Proof. The first assertion follows from Lemma 13.1. The last assertion follows from the first assertion.

In (12.3) we displayed a direct sum decomposition of $\tilde{H}_{q}$. For each summand we now consider the corresponding projection map.

Definition 13.3. For $\mu \in\{1, X, Y, C\}$ define an $\mathbb{F}$-linear map $P_{\mu}: \tilde{H}_{q} \rightarrow \tilde{H}_{q}$ such that $P_{\mu}$ acts as the identity on $\langle A\rangle \mu\langle B\rangle \mathbb{T}$, and as 0 on the other three summands in (12.3). Thus $P_{\mu}$ is the projection from $\tilde{H}_{q}$ onto $\langle A\rangle \mu\langle B\rangle \mathbb{T}$. For $h \in \tilde{H}_{q}$ we have

$$
P_{\mu}(A h)=A P_{\mu}(h), \quad P_{\mu}(h B)=P_{\mu}(h) B, \quad P_{\mu}(h v)=P_{\mu}(h) v, \quad \forall v \in \mathbb{T} .
$$

Moreover

$$
h=P_{1}(h)+P_{X}(h)+P_{Y}(h)+P_{C}(h) .
$$

For $h \in \tilde{H}_{q}$ we now consider how the projections $P_{\mu}(h)$ are related to the projections $\pi_{\nu}(h)$ from Definition 11.5. 
Lemma 13.4. Let $h$ denote an element of $\tilde{H}_{q}$, and write

$$
P_{C}(h)=\sum_{i, j \in \mathbb{N}} A^{i} C B^{j} t_{i j}, \quad t_{i j} \in \mathbb{T} .
$$

Then

$$
\begin{aligned}
& \pi_{1}(h)=P_{1}(h)+\sum_{i, j \in \mathbb{N}} A^{i} \pi_{1}(C) B^{j} t_{i j}, \\
& \pi_{X}(h)=P_{X}(h)+\sum_{i, j \in \mathbb{N}} A^{i} \pi_{X}(C) B^{j} t_{i j}, \\
& \pi_{Y}(h)=P_{Y}(h)+\sum_{i, j \in \mathbb{N}} A^{i} \pi_{Y}(C) B^{j} t_{i j}, \\
& \pi_{Y X}(h)=q^{-1} \sum_{i, j \in \mathbb{N}} A^{i} Y X B^{j} t_{i j}\left(1-t_{0}^{-2}\right) .
\end{aligned}
$$

Proof. We have both

$$
\begin{aligned}
& h=\pi_{1}(h)+\pi_{X}(h)+\pi_{Y}(h)+\pi_{Y X}(h), \\
& h=P_{1}(h)+P_{X}(h)+P_{Y}(h)+P_{C}(h) .
\end{aligned}
$$

In (13.5) eliminate $P_{C}(h)$ using (13.3), and evaluate the result using

$$
C=\pi_{1}(C)+\pi_{X}(C)+\pi_{Y}(C)+\pi_{Y X}(C) .
$$

By Lemma 11.6 we have $\pi_{Y X}(C)=q^{-1} Y X\left(1-t_{0}^{-2}\right)$. Subtracting (13.5) from (13.4) and using the above comments, we obtain

$$
\begin{aligned}
0= & \pi_{1}(h)-P_{1}(h)-\sum_{i, j \in \mathbb{N}} A^{i} \pi_{1}(C) B^{j} t_{i j} \\
& +\pi_{X}(h)-P_{X}(h)-\sum_{i, j \in \mathbb{N}} A^{i} \pi_{X}(C) B^{j} t_{i j} \\
& +\pi_{Y}(h)-P_{Y}(h)-\sum_{i, j \in \mathbb{N}} A^{i} \pi_{Y}(C) B^{j} t_{i j} \\
& +\pi_{Y X}(h)-q^{-1} \sum_{i, j \in \mathbb{N}} A^{i} Y X B^{j} t_{i j}\left(1-t_{0}^{-2}\right) .
\end{aligned}
$$

The elements (13.6), (13.7), (13.8), (13.9) are contained in the subspaces

$$
\langle A\rangle\langle B\rangle \mathbb{T}, \quad\langle A\rangle X\langle B\rangle \mathbb{T}, \quad\langle A\rangle Y\langle B\rangle \mathbb{T}, \quad\langle A\rangle Y X\langle B\rangle \mathbb{T}
$$

respectively. The sum of these subspaces is direct, so each of $(13.6),(13.7),(13.8),(13.9)$ is zero. The result follows.

Lemma 13.5. For $h \in \hat{H}_{q}$ the following are equivalent:

(i) $h \in\langle A, B, C, \mathbb{T}\rangle$.

(ii) $h\left(t_{0}-t_{0}^{-1}\right) \in\langle A, B, C, \mathbb{T}\rangle$. 
Proof. $(i) \Rightarrow(i i)$ Since $t_{0}-t_{0}^{-1} \in \mathbb{T}$.

$($ ii $) \Rightarrow(i)$ Observe by $(12.1)$ that $h\left(t_{0}-t_{0}^{-1}\right) \in \tilde{H}_{q}$. Write

$$
P_{C}\left(h\left(t_{0}-t_{0}^{-1}\right)\right)=\sum_{i, j \in \mathbb{N}} A^{i} C B^{j} t_{i j}, \quad t_{i j} \in \mathbb{T} .
$$

We first show that $h \in \tilde{H}_{q}$. Comparing (10.6) and (12.3) we find

$$
P_{X}\left(h\left(t_{0}-t_{0}^{-1}\right)\right)=0, \quad P_{Y}\left(h\left(t_{0}-t_{0}^{-1}\right)\right)=0 .
$$

By the equation on the right in (11.2),

$$
\pi_{\nu}\left(h\left(t_{0}-t_{0}^{-1}\right)\right)=\pi_{\nu}(h)\left(t_{0}-t_{0}^{-1}\right), \quad \nu \in\{1, X, Y, Y X\} .
$$

By this and Lemma 13.4,

$$
\pi_{Y X}(h)\left(t_{0}-t_{0}^{-1}\right)=\pi_{Y X}\left(h\left(t_{0}-t_{0}^{-1}\right)\right)=q^{-1} \sum_{i, j \in \mathbb{N}} A^{i} Y X B^{j} t_{i j}\left(1-t_{0}^{-2}\right) .
$$

By this and Lemma 8.9,

$$
\pi_{Y X}(h)=q^{-1} \sum_{i, j \in \mathbb{N}} A^{i} Y X B^{j} t_{i j} t_{0}^{-1} .
$$

In order to show that $h \in \tilde{H}_{q}$ we show that $t_{0}-t_{0}^{-1}$ divides $t_{i j}$ for all $i, j \in \mathbb{N}$. Observe

$$
\begin{aligned}
\pi_{Y}(h)\left(t_{0}-t_{0}^{-1}\right) & =\pi_{Y}\left(h\left(t_{0}-t_{0}^{-1}\right)\right) \stackrel{\text { by Lemma } 13.4}{=} \sum_{i, j \in \mathbb{N}} A^{i} \pi_{Y}(C) B^{j} t_{i j} \\
& \stackrel{\text { by Lemma } 11.6}{=} q^{-1} \sum_{i, j \in \mathbb{N}} A^{i}\left(Y t_{0}^{-1} T_{3}-Y B\right) B^{j} t_{i j} \\
& =q^{-1} \sum_{r, s \in \mathbb{N}} A^{r} Y B^{s}\left(t_{r s} t_{0}^{-1} T_{3}-t_{r, s-1}\right),
\end{aligned}
$$

where $t_{r,-1}=0$ for $r \in \mathbb{N}$. From this we see that $t_{0}-t_{0}^{-1}$ divides $t_{r s} t_{0}^{-1} T_{3}-t_{r, s-1}$ for all $r, s \in \mathbb{N}$. By this and induction on $s$ we find $t_{0}-t_{0}^{-1}$ divides $t_{r s}$ for all $r, s \in \mathbb{N}$. In other words, for all $r, s \in \mathbb{N}$ there exists $t_{r s}^{\prime} \in \mathbb{T}$ such that $t_{r s}=t_{r s}^{\prime}\left(t_{0}-t_{0}^{-1}\right)$. Now using (13.11),

$$
\pi_{Y X}(h)=q^{-1} \sum_{i, j \in \mathbb{N}} A^{i} Y X B^{j} t_{i j}^{\prime}\left(1-t_{0}^{-2}\right) \in\langle A\rangle Y X\langle B\rangle \mathbb{T}\left(1-t_{0}^{-2}\right) .
$$

By this and (12.1) we find $h \in \tilde{H}_{q}$. By the equation on the right in (13.2) and the equation on the left in (13.10),

$$
P_{X}(h)\left(t_{0}-t_{0}^{-1}\right)=P_{X}\left(h\left(t_{0}-t_{0}^{-1}\right)\right)=0 .
$$

Therefore $P_{X}(h)=0$ in view of Lemma 8.9. Similarly

$$
P_{Y}(h)\left(t_{0}-t_{0}^{-1}\right)=P_{Y}\left(h\left(t_{0}-t_{0}^{-1}\right)\right)=0,
$$

so $P_{Y}(h)=0$. Now

$$
\begin{aligned}
h= & P_{1}(h)+P_{X}(h)+P_{Y}(h)+P_{C}(h)=P_{1}(h)+P_{C}(h) \\
& \in\langle A\rangle\langle B\rangle \mathbb{T}+\langle A\rangle C\langle B\rangle \mathbb{T}=\langle A, B, C, \mathbb{T}\rangle .
\end{aligned}
$$

The result follows. 
Roughly speaking, the following result amounts to a universal analog of [11, Theorem 5.1].

Theorem 13.6. We have

$$
\langle A, B, C, \mathbb{T}\rangle=\left\{h \in \hat{H}_{q} \mid t_{0} h=h t_{0}\right\} .
$$

Proof. In (13.12) the inclusion $\subseteq$ holds by Lemma 6.4 and since $\left\{T_{i}\right\}_{i=1}^{3}$ are central in $\hat{H}_{q}$. We now obtain the inclusion $\supseteq$. Pick $h \in \hat{H}_{q}$ such that $t_{0} h=h t_{0}$. We show $h \in\langle A, B, C, \mathbb{T}\rangle$. By assumption $t_{0} h=h t_{0}$ so $h\left(t_{0}-t_{0}^{-1}\right)=t_{0} h-h t_{0}^{-1}$. By Lemma $13.2 t_{0} h-h t_{0}^{-1} \in\langle A, B, C, \mathbb{T}\rangle$. By these comments $h\left(t_{0}-t_{0}^{-1}\right) \in\langle A, B, C, \mathbb{T}\rangle$. By this and Lemma $13.5 h \in\langle A, B, C, \mathbb{T}\rangle$.

\section{A presentation for the algebra $\langle A, B, C, \mathbb{T}\rangle$}

We continue to discuss the subalgebra $\langle A, B, C, \mathbb{T}\rangle$ of $\hat{H}_{q}$. In this section we give a presentation for $\langle A, B, C, \mathbb{T}\rangle$ by generators and relations. Roughly speaking, this presentation amounts to a $q$-analog of [13, Theorem 2.1] and a universal analog of [10, Definition 6.1, Corollary 6.3].

Theorem 14.1. The $\mathbb{F}$-algebra $\langle A, B, C, \mathbb{T}\rangle$ is presented by generators and relations in the following way. The generators are $A, B, C, t_{0}^{ \pm 1},\left\{T_{i}\right\}_{i=1}^{3}$. The relations assert that each of $t_{0}^{ \pm 1}$, $\left\{T_{i}\right\}_{i=1}^{3}$ is central and $t_{0} t_{0}^{-1}=1, t_{0}^{-1} t_{0}=1$,

$$
\begin{aligned}
& A+\frac{q B C-q^{-1} C B}{q^{2}-q^{-2}}=\frac{\alpha}{q+q^{-1}}, \\
& B+\frac{q C A-q^{-1} A C}{q^{2}-q^{-2}}=\frac{\beta}{q+q^{-1}}, \\
& C+\frac{q A B-q^{-1} B A}{q^{2}-q^{-2}}=\frac{\gamma}{q+q^{-1}}, \\
& q^{-1} A C B+q^{-2} A^{2}+q^{-2} B^{2}+q^{2} C^{2}-q^{-1} A \alpha-q^{-1} B \beta-q C \gamma \\
& \quad=\left(q+q^{-1}\right)^{2}-\left(q^{-1} t_{0}+q t_{0}^{-1}\right)^{2}-T_{1}^{2}-T_{2}^{2}-T_{3}^{2}-\left(q^{-1} t_{0}+q t_{0}^{-1}\right) T_{1} T_{2} T_{3},
\end{aligned}
$$

where

$$
\begin{aligned}
& \alpha=\left(q^{-1} t_{0}+q t_{0}^{-1}\right) T_{1}+T_{2} T_{3}, \quad \beta=\left(q^{-1} t_{0}+q t_{0}^{-1}\right) T_{3}+T_{1} T_{2}, \\
& \gamma=\left(q^{-1} t_{0}+q t_{0}^{-1}\right) T_{2}+T_{3} T_{1} .
\end{aligned}
$$

Proof. Let $\mathcal{A}_{q}$ denote the $\mathbb{F}$-algebra defined by generators $A, B, C, t_{0}^{ \pm 1},\left\{T_{i}\right\}_{i=1}^{3}$ and the above relations. Since these relations hold in $\hat{H}_{q}$ there exists an $\mathbb{F}$-algebra homomorphism $\mathcal{A}_{q} \rightarrow \hat{H}_{q}$ that sends each generator $A, B, C, t_{0}^{ \pm 1},\left\{T_{i}\right\}_{i=1}^{3}$ of $\mathcal{A}_{q}$ to the corresponding element in $\hat{H}_{q}$. Under this homomorphism the image of $\mathcal{A}_{q}$ is the subalgebra $\langle A, B, C, \mathbb{T}\rangle$ of $\hat{H}_{q}$. We show that the homomorphism is injective. To this end, we claim that the following vectors span the $\mathbb{F}$-vector space $\mathcal{A}_{q}$ :

$$
A^{i} C^{j} B^{k} t_{0}^{\ell} T_{1}^{r} T_{2}^{s} T_{3}^{t}, \quad j \in\{0,1\}, \quad \ell \in \mathbb{Z}, \quad i, k, r, s, t \in \mathbb{N} .
$$

To prove the claim, note that the elements $A, B, C$ of $\mathcal{A}_{q}$ satisfy the defining relations for $\Delta_{q}$ given in Definition 2.1. Therefore there exists an $\mathbb{F}$-algebra homomorphism $\Delta_{q} \rightarrow \mathcal{A}_{q}$ that sends each generator $A, B, C$ of $\Delta_{q}$ to the corresponding element in $\mathcal{A}_{q}$. In (2.6) we displayed a basis for the $\mathbb{F}$-vector space $\Delta_{q}$. When our homomorphism $\Delta_{q} \rightarrow \mathcal{A}_{q}$ is applied to a vector in this basis, the image is contained in the span of (14.1). Therefore the span of (14.1) contains the subalgebra of $\mathcal{A}_{q}$ generated by $A, B, C$. By construction $\mathcal{A}_{q}$ is generated by $A, B, C, t_{0}^{ \pm 1}$, $\left\{T_{i}\right\}_{i=1}^{3}$. By definition each element $A, B, C$ of $\mathcal{A}_{q}$ commutes with each element $t_{0}^{ \pm 1},\left\{T_{i}\right\}_{i=1}^{3}$ 
of $\mathcal{A}_{q}$. By construction the span of (14.1) is closed under multiplication by each element $t_{0}^{ \pm 1}$, $\left\{T_{i}\right\}_{i=1}^{3}$ of $\mathcal{A}_{q}$. By these comments the vectors (14.1) span $\mathcal{A}_{q}$. The claim is proven. When we apply our homomorphism $\mathcal{A}_{q} \rightarrow \hat{H}_{q}$ to the vectors (14.1), we get the basis for $\langle A, B, C, \mathbb{T}\rangle$ given in Proposition 12.13. Therefore the vectors (14.1) form a basis for $\mathcal{A}_{q}$ and our homomorphism $\mathcal{A}_{q} \rightarrow \hat{H}_{q}$ is injective. The result follows.

\section{The center of $\hat{H}_{q}$}

In this section we describe the center $Z\left(\hat{H}_{q}\right)$.

Recall that the $\left\{T_{i}\right\}_{i \in \mathbb{I}}$ are central in $\hat{H}_{q}$. We are going to show that $\left\{T_{i}\right\}_{i \in \mathbb{I}}$ generate $Z\left(\hat{H}_{q}\right)$, provided that $q$ is not a root of unity. In this derivation we will repeatedly use the basis for $\hat{H}_{q}$ given in Proposition 7.8.

Definition 15.1. Let $K$ denote the 2 -sided ideal of $\hat{H}_{q}$ generated by $\left\{T_{i}\right\}_{i \in \mathbb{I}}$. Thus

$$
K=\sum_{i \in \mathbb{I}} \hat{H}_{q} T_{i}
$$

Lemma 15.2. The following is a basis for the $\mathbb{F}$-vector space $K$ :

$$
Y^{i} X^{j} t_{0}^{k} T_{0}^{\ell} T_{1}^{r} T_{2}^{s} T_{3}^{t}, \quad i, j \in \mathbb{Z}, \quad k \in\{0,1\}, \quad \ell, r, s, t \in \mathbb{N}, \quad(\ell, r, s, t) \neq(0,0,0,0) .
$$

Proof. Use Proposition 7.8.

Lemma 15.3. The following is a basis for a complement of $K$ in $\hat{H}_{q}$ :

$$
Y^{i} X^{j} t_{0}^{k}, \quad i, j \in \mathbb{Z}, \quad k \in\{0,1\} .
$$

Proof. Compare Proposition 7.8 and Lemma 15.2.

Definition 15.4. Let $\bar{H}_{q}$ denote the quotient $\mathbb{F}$-algebra $\bar{H}_{q}=\hat{H}_{q} / K$. Recall that the canonical map $\hat{H}_{q} \rightarrow \bar{H}_{q}$ is a surjective $\mathbb{F}$-algebra homomorphism with kernel $K$. For $h \in \hat{H}_{q}$ let $\bar{h}$ denote the image of $h$ under this map. By construction $\bar{T}_{i}=0$ for $i \in \mathbb{I}$.

Lemma 15.5. The following is a basis for the $\mathbb{F}$-vector space $\bar{H}_{q}$ :

$$
\bar{Y}^{i} \bar{X}^{j} \bar{t}_{0}^{k}, \quad i, j \in \mathbb{Z}, \quad k \in\{0,1\} .
$$

Proof. Use Lemma 15.3.

Lemma 15.6. Referring to Definition 5.12 we have $\bar{C}_{i}=0$ for $i \in \mathbb{I}$.

Proof. By Proposition 5.14 and since $\bar{T}_{j}=0$ for $j \in \mathbb{I}$.

Lemma 15.7. The following relations hold in $\bar{H}_{q}$ :

$$
\begin{array}{ll}
\bar{X} \bar{Y}=q^{2} \bar{Y} \bar{X}, & \bar{t}_{0}^{2}=-1, \\
\bar{t}_{0} \bar{X}=\bar{X}^{-1} \bar{t}_{0}, & \bar{t}_{0} \bar{Y}=\bar{Y}^{-1} \bar{t}_{0} .
\end{array}
$$

Proof. The equation on the left in (15.1) follows from Definition 5.12 and Lemma 15.6. To get the equation on the right in (15.1), apply the map $h \mapsto \bar{h}$ to each side of $t_{0}^{2}=t_{0} T_{0}-1$. To get the equations in (15.2), apply the map $h \mapsto \bar{h}$ to each side of (5.4) and (5.6). 
Definition 15.8. We endow the set $\mathbb{N}^{4}$ with a partial order $\leq$ as follows. Let $\left(n_{0}, n_{1}, n_{2}, n_{3}\right)$ and $\left(n_{0}^{\prime}, n_{1}^{\prime}, n_{2}^{\prime}, n_{3}^{\prime}\right)$ denote elements of $\mathbb{N}^{4}$. Then $\left(n_{0}, n_{1}, n_{2}, n_{3}\right) \leq\left(n_{0}^{\prime}, n_{1}^{\prime}, n_{2}^{\prime}, n_{3}^{\prime}\right)$ whenever $n_{i} \leq n_{i}^{\prime}$ for $0 \leq i \leq 3$.

We have some comments. Fix $(\ell, r, s, t) \in \mathbb{N}^{4}$ and define

$$
L=\hat{H}_{q} T_{0}^{\ell} T_{1}^{r} T_{2}^{s} T_{3}^{t}
$$

Then $L$ is a 2 -sided ideal of $\hat{H}_{q}$ with basis

$$
\begin{aligned}
& Y^{i} X^{j} t_{0}^{k} T_{0}^{\ell^{\prime}} T_{1}^{r^{\prime}} T_{2}^{s^{\prime}} T_{3}^{t^{\prime}}, \quad i, j \in \mathbb{Z}, \quad k \in\{0,1\}, \\
& \left(\ell^{\prime}, r^{\prime}, s^{\prime}, t^{\prime}\right) \in \mathbb{N}^{4}, \quad(\ell, r, s, t) \leq\left(\ell^{\prime}, r^{\prime}, s^{\prime}, t^{\prime}\right) .
\end{aligned}
$$

Observe that $K L$ is a 2 -sided ideal of $\hat{H}_{q}$ with basis

$$
\begin{aligned}
& Y^{i} X^{j} t_{0}^{k} T_{0}^{\ell^{\prime}} T_{1}^{r^{\prime}} T_{2}^{s^{\prime}} T_{3}^{t^{\prime}}, \quad i, j \in \mathbb{Z}, \quad k \in\{0,1\}, \\
& \left(\ell^{\prime}, r^{\prime}, s^{\prime}, t^{\prime}\right) \in \mathbb{N}^{4}, \quad(\ell, r, s, t)<\left(\ell^{\prime}, r^{\prime}, s^{\prime}, t^{\prime}\right) .
\end{aligned}
$$

Define

$$
R=\hat{H}_{q} T_{0}^{\ell+1}+\hat{H}_{q} T_{1}^{r+1}+\hat{H}_{q} T_{2}^{s+1}+\hat{H}_{q} T_{3}^{t+1} .
$$

Then $R$ is a 2 -sided ideal of $\hat{H}_{q}$ with basis

$$
\begin{aligned}
& Y^{i} X^{j} t_{0}^{k} T_{0}^{\ell^{\prime}} T_{1}^{r^{\prime}} T_{2}^{s^{\prime}} T_{3}^{t^{\prime}}, \quad i, j \in \mathbb{Z}, \quad k \in\{0,1\}, \\
& \left(\ell^{\prime}, r^{\prime}, s^{\prime}, t^{\prime}\right) \in \mathbb{N}^{4}, \quad\left(\ell^{\prime}, r^{\prime}, s^{\prime}, t^{\prime}\right) \not \leq(\ell, r, s, t) .
\end{aligned}
$$

Comparing the above bases we find

$$
L \cap R=K L .
$$

Theorem 15.9. Assume that $q$ is not a root of unity. Then the $\mathbb{F}$-algebra $Z\left(\hat{H}_{q}\right)$ is generated by $\left\{T_{i}\right\}_{i \in \mathbb{I}}$.

Proof. Consider the subalgebra $\left\langle T_{0}, T_{1}, T_{2}, T_{3}\right\rangle$ of $\hat{H}_{q}$. This subalgebra is contained in $Z\left(\hat{H}_{q}\right)$. We assume that the containment is proper, and obtain a contradiction. Pick

$$
h \in Z\left(\hat{H}_{q}\right), \quad h \notin\left\langle T_{0}, T_{1}, T_{2}, T_{3}\right\rangle .
$$

In view of Proposition 7.8 we write

$$
h=\sum_{\ell, r, s, t \in \mathbb{N}} h_{\ell, r, s, t} T_{0}^{\ell} T_{1}^{r} T_{2}^{s} T_{3}^{t}, \quad h_{\ell, r, s, t} \in\left\langle Y^{ \pm 1}\right\rangle\left\langle X^{ \pm 1}\right\rangle+\left\langle Y^{ \pm 1}\right\rangle\left\langle X^{ \pm 1}\right\rangle t_{0} .
$$

Define the set

$$
S(h)=\left\{(\ell, r, s, t) \in \mathbb{N}^{4} \mid h_{\ell, r, s, t} \neq 0\right\} .
$$

By construction the cardinality $|S(h)|$ is finite. Without loss of generality, we assume that $h$ has been chosen such that $|S(h)|$ is minimal subject to (15.6). Note that $h \neq 0$ so $S(h)$ is nonempty. There exists an element of $S(h)$ that is not greater than any other element of $S(h)$, with respect to the partial order $\leq$ from Definition 15.8. Denote this element by $(\ell, r, s, t)$. We will be discussing the corresponding ideals $L, R$ of $\hat{H}_{q}$ from (15.3) and (15.4). By construction

$$
h-h_{\ell, r, s, t} T_{0}^{\ell} T_{1}^{r} T_{2}^{s} T_{3}^{t} \in R .
$$


Write

$$
h_{\ell, r, s, t}=\sum_{i, j \in \mathbb{Z}} \alpha_{i j} Y^{i} X^{j}+\sum_{i, j \in \mathbb{Z}} \beta_{i j} Y^{i} X^{j} t_{0}, \quad \alpha_{i j}, \beta_{i j} \in \mathbb{F} .
$$

We take the commutator of (15.7) with each of $X, Y$. We start with $X$. The ideal $R$ contains

$$
X h-h X-\left(X h_{\ell, r, s, t}-h_{\ell, r, s, t} X\right) T_{0}^{\ell} T_{1}^{r} T_{2}^{s} T_{3}^{t} .
$$

By assumption $h \in Z\left(\hat{H}_{q}\right)$ so $X h-h X=0$. Therefore $R$ contains

$$
\left(X h_{\ell, r, s, t}-h_{\ell, r, s, t} X\right) T_{0}^{\ell} T_{1}^{r} T_{2}^{s} T_{3}^{t} .
$$

The element (15.9) is contained in $L$ by (15.3). By these comments and (15.5), the element (15.9) is contained in $K L$. By Lemma 8.9 the map $\hat{H}_{q} \rightarrow L, g \mapsto g T_{0}^{\ell} T_{1}^{r} T_{2}^{s} T_{3}^{t}$ is a bijection. Under this map the image of $K$ is $K L$. Therefore in (15.9), the expression in parenthesis is contained in $K$. In other words, in the notation of Definition 15.4,

$$
\bar{X} \bar{h}_{\ell, r, s, t}-\bar{h}_{\ell, r, s, t} \bar{X}=0 .
$$

Expanding (15.10) using (15.8) we obtain

$$
0=\sum_{i, j \in \mathbb{Z}} \alpha_{i j}\left(\bar{X} \bar{Y}^{i} \bar{X}^{j}-\bar{Y}^{i} \bar{X}^{j} \bar{X}\right)+\sum_{i, j \in \mathbb{Z}} \beta_{i j}\left(\bar{X} \bar{Y}^{i} \bar{X}^{j} \bar{t}_{0}-\bar{Y}^{i} \bar{X}^{j} \bar{t}_{0} \bar{X}\right) .
$$

Simplifying this using Lemma 15.7 we obtain

$$
0=\sum_{i, j \in \mathbb{Z}} \alpha_{i j} \bar{Y}^{i} \bar{X}^{j+1}\left(q^{2 i}-1\right)+\sum_{i, j \in \mathbb{Z}} \beta_{i j}\left(\bar{Y}^{i} \bar{X}^{j+1} \bar{t}_{0} q^{2 i}-\bar{Y}^{i} \bar{X}^{j-1} \bar{t}_{0}\right) .
$$

Adjusting the indices $i, j$ in the above sums,

$$
0=\sum_{i, j \in \mathbb{Z}} \bar{Y}^{i} \bar{X}^{j} \alpha_{i, j-1}\left(q^{2 i}-1\right)+\sum_{i, j \in \mathbb{Z}} \bar{Y}^{i} \bar{X}^{j} \bar{t}_{0}\left(\beta_{i, j-1} q^{2 i}-\beta_{i, j+1}\right) .
$$

By this and Lemma 15.5 we find

$$
\begin{aligned}
& \alpha_{i, j-1}\left(q^{2 i}-1\right)=0, \quad i, j \in \mathbb{Z}, \\
& \beta_{i, j-1} q^{2 i}-\beta_{i, j+1}=0, \quad i, j \in \mathbb{Z} .
\end{aligned}
$$

Taking the commutator of (15.7) with $Y$, we similarly obtain

$$
\begin{aligned}
& \alpha_{i-1, j}\left(q^{2 j}-1\right)=0, \quad i, j \in \mathbb{Z}, \\
& \beta_{i-1, j}-\beta_{i+1, j} q^{-2 j}=0, \quad i, j \in \mathbb{Z} .
\end{aligned}
$$

By (15.11), (15.13) and since $q$ is not a root of unity,

$$
\alpha_{i j}=0 \quad \text { if } \quad(i, j) \neq(0,0), \quad i, j \in \mathbb{Z} .
$$

By (15.12) or (15.14), and since finitely many of the $\beta_{i j}$ are nonzero,

$$
\beta_{i j}=0, \quad i, j \in \mathbb{Z}
$$

Evaluating (15.8) using these comments we obtain $h_{\ell, r, s, t}=\alpha_{00} \in \mathbb{F}$. Define

$$
h^{\prime}=h-h_{\ell, r, s, t} T_{0}^{\ell} T_{1}^{r} T_{2}^{s} T_{3}^{t} .
$$


We have two comments about $h^{\prime}$. First of all,

$$
h-h^{\prime} \in\left\langle T_{0}, T_{1}, T_{2}, T_{3}\right\rangle \subseteq Z\left(\hat{H}_{q}\right),
$$

so

$$
h^{\prime} \in Z\left(\hat{H}_{q}\right), \quad h^{\prime} \notin\left\langle T_{0}, T_{1}, T_{2}, T_{3}\right\rangle .
$$

Second of all, $S\left(h^{\prime}\right)$ is obtained from $S(h)$ by deleting the element $(\ell, r, s, t)$; therefore $\left|S\left(h^{\prime}\right)\right|=$ $|S(h)|-1$. These two comments contradict the minimality of $|S(h)|$. The result follows.

Roughly speaking, the following two corollaries amount to a universal analog of [11, Theorem 5.3].

Corollary 15.10. Assume that $q$ is not a root of unity. Then the following is a basis for the $\mathbb{F}$-vector space $Z\left(\hat{H}_{q}\right)$ :

$$
T_{0}^{\ell} T_{1}^{r} T_{2}^{s} T_{3}^{t}, \quad \ell, r, s, t \in \mathbb{N} .
$$

Proof. The vectors (15.15) span $Z\left(\hat{H}_{q}\right)$ by Theorem 15.9. The vectors (15.15) are linearly independent because they are included in the linearly independent set (7.6).

Corollary 15.11. Assume that $q$ is not a root of unity. Then there exists an isomorphism of $\mathbb{F}$-algebras $Z\left(\hat{H}_{q}\right) \rightarrow \mathbb{F}\left[\lambda_{0}, \lambda_{1}, \lambda_{2}, \lambda_{3}\right]$ that sends $T_{i} \mapsto \lambda_{i}$ for $0 \leq i \leq 3$.

Proof. Immediate from Corollary 15.10.

\section{Discussion}

In this section we compare our main results with the results of Koornwinder [10, 11].

Recall from Definition 3.1 that $\hat{H}_{q}$ is the universal DAHA of type $\left(C_{1}^{\vee}, C_{1}\right)$. In [10, 11] Koornwinder works with a related algebra $\tilde{\mathfrak{H}}$ called the DAHA of type $\left(C_{1}^{\vee}, C_{1}\right)$. We will compare these algebras shortly. Recall the set $\mathbb{I}=\{0,1,2,3\}$. By Lemma 5.2 the $\mathbb{F}$-algebra $\hat{H}_{q}$ has a presentation by generators $\left\{t_{i}\right\}_{i \in \mathbb{I}},\left\{T_{i}\right\}_{i \in \mathbb{I}}$ and relations

$$
\begin{aligned}
& t_{i}^{2}=T_{i} t_{i}-1, \quad i \in \mathbb{I}, \\
& T_{i} \text { is central, } \\
& t_{0} t_{1} t_{2} t_{3}=q^{-1} .
\end{aligned}
$$

Definition 16.1. Let $\left\{P_{i}\right\}_{i \in \mathbb{I}}$ denote scalars in $\mathbb{F}$. Define an $\mathbb{F}$-algebra $\hat{H}_{q}\left(P_{0}, P_{1}, P_{2}, P_{3}\right)$ by generators $\left\{t_{i}\right\}_{i \in \mathbb{I}}$ and relations

$$
t_{i}^{2}=P_{i} t_{i}-1, \quad i \in \mathbb{I}, \quad t_{0} t_{1} t_{2} t_{3}=q^{-1} .
$$

Lemma 16.2. For $i \in \mathbb{I}$ the element $t_{i}$ of $\hat{H}_{q}\left(P_{0}, P_{1}, P_{2}, P_{3}\right)$ is invertible and $t_{i}+t_{i}^{-1}=P_{i}$.

By construction there exists a unique $\mathbb{F}$-algebra homomorphism $\hat{H}_{q} \rightarrow \hat{H}_{q}\left(P_{0}, P_{1}, P_{2}, P_{3}\right)$ that sends $t_{i} \mapsto t_{i}$ and $T_{i} \mapsto P_{i}$ for $i \in \mathbb{I}$. This map is surjective. We denote this map by $\varepsilon\left(P_{0}, P_{1}, P_{2}, P_{3}\right)$.

Recall the elements $A, B, C$ of $\hat{H}_{q}$ :

$$
\begin{aligned}
& A=t_{1} t_{0}+\left(t_{1} t_{0}\right)^{-1}=t_{0} t_{1}+\left(t_{0} t_{1}\right)^{-1}, \quad B=t_{3} t_{0}+\left(t_{3} t_{0}\right)^{-1}=t_{0} t_{3}+\left(t_{0} t_{3}\right)^{-1}, \\
& C=t_{2} t_{0}+\left(t_{2} t_{0}\right)^{-1}=t_{0} t_{2}+\left(t_{0} t_{2}\right)^{-1} .
\end{aligned}
$$

We retain the notation $A, B, C$ for their images under $\varepsilon\left(P_{0}, P_{1}, P_{2}, P_{3}\right)$. Recall from Definition 7.10 the subalgebra $\mathbb{T}=\left\langle t_{0}^{ \pm 1}, T_{1}, T_{2}, T_{3}\right\rangle$ of $\hat{H}_{q}$. By $(5.1), \mathbb{T}=\left\langle t_{0}, T_{0}, T_{1}, T_{2}, T_{3}\right\rangle$. The subalgebra $\langle A, B, C, \mathbb{T}\rangle$ of $\hat{H}_{q}$ was discussed in Propositions 12.12, 12.13 and Theorems 13.6, 14.1. 
Definition 16.3. Consider the subalgebra $\langle A, B, C, \mathbb{T}\rangle$ of $\hat{H}_{q}$. Let $\mathcal{A}$ denote the image of $\langle A, B, C, \mathbb{T}\rangle$ under the map $\varepsilon\left(P_{0}, P_{1}, P_{2}, P_{3}\right)$. Thus $\mathcal{A}$ is the subalgebra of $\hat{H}_{q}\left(P_{0}, P_{1}, P_{2}, P_{3}\right)$ generated by $A, B, C, t_{0}$.

Proposition 16.4. The $\mathbb{F}$-algebra $\mathcal{A}$ is presented by generators and relations in the following way. The generators are $A, B, C, t_{0}$. The relations assert that $t_{0}$ is central and $t_{0}^{2}=P_{0} t_{0}-1$,

$$
\begin{aligned}
& A+\frac{q B C-q^{-1} C B}{q^{2}-q^{-2}}=\frac{\alpha}{q+q^{-1}}, \\
& B+\frac{q C A-q^{-1} A C}{q^{2}-q^{-2}}=\frac{\beta}{q+q^{-1}}, \\
& \begin{array}{l}
C+\frac{q A B-q^{-1} B A}{q^{2}-q^{-2}}=\frac{\gamma}{q+q^{-1}}, \\
q^{-1} A C B+q^{-2} A^{2}+q^{-2} B^{2}+q^{2} C^{2}-q^{-1} A \alpha-q^{-1} B \beta-q C \gamma \\
\quad=\left(q+q^{-1}\right)^{2}-\left(q^{-1} t_{0}+q t_{0}^{-1}\right)^{2}-P_{1}^{2}-P_{2}^{2}-P_{3}^{2}-\left(q^{-1} t_{0}+q t_{0}^{-1}\right) P_{1} P_{2} P_{3},
\end{array}
\end{aligned}
$$

where

$$
\begin{array}{ll}
\alpha=\left(q^{-1} t_{0}+q t_{0}^{-1}\right) P_{1}+P_{2} P_{3}, & \beta=\left(q^{-1} t_{0}+q t_{0}^{-1}\right) P_{3}+P_{1} P_{2}, \\
\gamma=\left(q^{-1} t_{0}+q t_{0}^{-1}\right) P_{2}+P_{3} P_{1}, & t_{0}^{-1}=P_{0}-t_{0} .
\end{array}
$$

Proof. In the relations of Theorem 14.1, first replace $t_{0}^{-1}$ by $T_{0}-t_{0}$ and then replace $T_{i}$ by $P_{i}$ for $i \in \mathbb{I}$.

By the first three displayed relations in Proposition 16.4, the $\mathbb{F}$-algebra $\mathcal{A}$ is generated by $t_{0}$ together with any two of $A, B, C$. We now give a presentation of $\mathcal{A}$ by generators and relations, using the generators $A, B, t_{0}$.

Proposition 16.5. The $\mathbb{F}$-algebra $\mathcal{A}$ is presented by generators $A, B$, $t_{0}$ and relations

$$
\begin{aligned}
& t_{0} A=A t_{0}, \quad t_{0} B=B t_{0}, \quad t_{0}^{2}=P_{0} t_{0}-1, \\
& \begin{aligned}
A^{2} B & -\left(q^{2}+q^{-2}\right) A B A+B A^{2}+\left(q^{2}-q^{-2}\right)^{2} B+\left(q-q^{-1}\right)^{2} A \gamma \\
\quad & \left(q-q^{-1}\right)\left(q^{2}-q^{-2}\right) \beta
\end{aligned} \\
& B^{2} A-\left(q^{2}+q^{-2}\right) B A B+A B^{2}+\left(q^{2}-q^{-2}\right)^{2} A+\left(q-q^{-1}\right)^{2} B \gamma \\
& \quad=\left(q-q^{-1}\right)\left(q^{2}-q^{-2}\right) \alpha, \\
& q^{-1} A \mathcal{C} B+q^{-2} A^{2}+q^{-2} B^{2}+q^{2} \mathcal{C}^{2}-q^{-1} A \alpha-q^{-1} B \beta-q \mathcal{C} \gamma \\
& \quad=\left(q+q^{-1}\right)^{2}-\left(q^{-1} t_{0}+q t_{0}^{-1}\right)^{2}-P_{1}^{2}-P_{2}^{2}-P_{3}^{2}-\left(q^{-1} t_{0}+q t_{0}^{-1}\right) P_{1} P_{2} P_{3},
\end{aligned}
$$

where

$$
\begin{array}{ll}
\mathcal{C}=\frac{\gamma}{q+q^{-1}}-\frac{q A B-q^{-1} B A}{q^{2}-q^{-2}}, & \alpha=\left(q^{-1} t_{0}+q t_{0}^{-1}\right) P_{1}+P_{2} P_{3}, \\
\beta=\left(q^{-1} t_{0}+q t_{0}^{-1}\right) P_{3}+P_{1} P_{2}, & \gamma=\left(q^{-1} t_{0}+q t_{0}^{-1}\right) P_{2}+P_{3} P_{1}, \quad t_{0}^{-1}=P_{0}-t_{0} .
\end{array}
$$

Proof. In the first two displayed relations of Proposition 16.4, eliminate $C$ using the third displayed relation.

We now bring in the work of Koornwinder [10, 11]. In [10, equations (3.1)-(3.4)] Koornwinder defines an algebra $\tilde{\mathfrak{H}}$. The definition involves some scalars $q, a, b, c, d$. For notational convenience we replace Koornwinder's $q, a, b, c, d$ by their squares. 
Definition 16.6 ([10, equations (3.1)-(3.4)]). Fix nonzero scalars $a, b, c, d$ in $\mathbb{F}$. The $\mathbb{F}$-algebra $\tilde{\mathfrak{H}}=\tilde{\mathfrak{H}}_{q}(a, b, c, d)$ is defined by generators $Z, Z^{-1}, \mathcal{T}_{1}, \mathcal{T}_{0}$ and relations

$$
\begin{array}{ll}
\left(\mathcal{T}_{1}+a^{2} b^{2}\right)\left(\mathcal{T}_{1}+1\right)=0, & \left(\mathcal{T}_{0}+q^{-2} c^{2} d^{2}\right)\left(\mathcal{T}_{0}+1\right)=0, \\
\left(\mathcal{T}_{1} Z+a^{2}\right)\left(\mathcal{T}_{1} Z+b^{2}\right)=0, & \left(q^{2} \mathcal{T}_{0} Z^{-1}+c^{2}\right)\left(q^{2} \mathcal{T}_{0} Z^{-1}+d^{2}\right)=0 .
\end{array}
$$

Lemma 16.7 ([10, equations $(3.5),(3.6)])$. The elements $\mathcal{T}_{1}, \mathcal{T}_{0}$ of $\tilde{\mathfrak{H}}$ are invertible and

$$
\mathcal{T}_{1}^{-1}=-a^{-2} b^{-2} \mathcal{T}_{1}-1-a^{-2} b^{-2}, \quad \mathcal{T}_{0}^{-1}=-q^{2} c^{-2} d^{-2} \mathcal{T}_{0}-1-q^{2} c^{-2} d^{-2} .
$$

From now on, assume

$$
\begin{array}{ll}
P_{0}=a b+a^{-1} b^{-1}, & P_{1}=a b^{-1}+a^{-1} b, \\
P_{2}=c d^{-1}+c^{-1} d, & P_{3}=q^{-1} c d+q c^{-1} d^{-1} .
\end{array}
$$

Lemma 16.8. There exists an isomorphism of $\mathbb{F}$-algebras

$$
\hat{H}_{q}\left(P_{0}, P_{1}, P_{2}, P_{3}\right) \rightarrow \tilde{\mathfrak{H}}_{q}(a, b, c, d)
$$

that sends

$$
t_{0} \mapsto-a^{-1} b^{-1} \mathcal{T}_{1}, \quad t_{1} \mapsto-a b \mathcal{T}_{1}^{-1} Z^{-1}, \quad t_{2} \mapsto-q^{-2} c d Z \mathcal{T}_{0}^{-1}, \quad t_{3} \mapsto-q c^{-1} d^{-1} \mathcal{T}_{0}
$$

The inverse isomorphism sends

$$
Z \mapsto q t_{2} t_{3}, \quad Z^{-1} \mapsto t_{0} t_{1}, \quad \mathcal{T}_{1} \mapsto-a b t_{0}, \quad \mathcal{T}_{0} \mapsto-q^{-1} c d t_{3} .
$$

Proof. One checks that the above maps are $\mathbb{F}$-algebra homomorphisms, and that they are inverses. Consequently they are isomorphisms.

From now on, we identify the $\mathbb{F}$-algebras $\hat{H}_{q}\left(P_{0}, P_{1}, P_{2}, P_{3}\right)$ and $\tilde{\mathfrak{H}}_{q}(a, b, c, d)$ via the isomorphism in Lemma 16.8 , and call the result $\tilde{\mathfrak{H}}$.

In [10, equations (3.8), (3.9)] Koornwinder discusses two elements of $\tilde{\mathfrak{H}}$. The first is $Y+$ $q^{-2} a^{2} b^{2} c^{2} d^{2} Y^{-1}$ where $Y=\mathcal{T}_{1} \mathcal{T}_{0}$. The second is $Z+Z^{-1}$. These elements are related to $A, B$ as follows.

Lemma 16.9. In the algebra $\tilde{\mathfrak{H}}$,

$$
Z+Z^{-1}=A, \quad Y+q^{-2} a^{2} b^{2} c^{2} d^{2} Y^{-1}=q^{-1} a b c d B .
$$

Proof. Use Lemma 16.8.

In [10, Definition 6.1] Koornwinder defines an $\mathbb{F}$-algebra $\widetilde{A W}\left(3, Q_{0}\right)$ by generators and relations. See also [11, Definition 2.5]. In [10, Corollary 6.3] Koornwinder displays an injection of $\mathbb{F}$-algebras $\widetilde{A W}\left(3, Q_{0}\right) \rightarrow \tilde{\mathfrak{H}}$. Consider the image of $\widetilde{A W}\left(3, Q_{0}\right)$ under this injection. By construction and Lemma 16.9 , this image is the subalgebra of $\tilde{\mathfrak{H}}$ generated by $A, B, t_{0}$. In other words, the image is $\mathcal{A}$. Thus [10, Definition 6.1, Corollary 6.3] yields a presentation of $\mathcal{A}$ by generators and relations, using the generators $A, B, t_{0}$. The presentation looks as follows in terms of $\left\{P_{i}\right\}_{i \in \mathbb{I}}$.

Theorem 16.10 ([10, Definition 6.1, Corollary 6.3]). The $\mathbb{F}$-algebra $\mathcal{A}$ is presented by generators $A, B, t_{0}$ and relations

$$
t_{0} A=A t_{0}, \quad t_{0} B=B t_{0}, \quad t_{0}^{2}=P_{0} t_{0}-1,
$$




$$
\begin{aligned}
& A^{2} B-\left(q^{2}+q^{-2}\right) A B A+B A^{2}+\left(q^{2}-q^{-2}\right)^{2} B+\left(q-q^{-1}\right)^{2} A \gamma=\left(q-q^{-1}\right)\left(q^{2}-q^{-2}\right) \beta, \\
& B^{2} A-\left(q^{2}+q^{-2}\right) B A B+A B^{2}+\left(q^{2}-q^{-2}\right)^{2} A+\left(q-q^{-1}\right)^{2} B \gamma=\left(q-q^{-1}\right)\left(q^{2}-q^{-2}\right) \alpha, \\
& \frac{A B A B}{\left(q^{2}-q^{-2}\right)^{2}}-\frac{B A B A\left(q^{4}+1+q^{-4}\right)}{\left(q^{2}-q^{-2}\right)^{2}}+\frac{B^{2} A^{2}\left(q^{2}+q^{-2}\right)}{\left(q^{2}-q^{-2}\right)^{2}}+A^{2}\left(q^{2}+q^{-2}\right)+B^{2}\left(q^{2}+q^{-2}\right) \\
& \quad+\frac{A B \gamma}{\left(q+q^{-1}\right)^{2}}+\frac{B A \gamma\left(q-q^{-1}\right)\left(q^{3}-q^{-3}\right)}{\left(q^{2}-q^{-2}\right)^{2}}-\frac{A \alpha\left(q^{3}-q^{-3}\right)}{q^{2}-q^{-2}}-\frac{B \beta\left(q^{3}-q^{-3}\right)}{q^{2}-q^{-2}}-\frac{\gamma^{2}}{\left(q+q^{-1}\right)^{2}} \\
& \quad=\left(q+q^{-1}\right)^{2}-\left(q^{-1} t_{0}+q t_{0}^{-1}\right)^{2}-P_{1}^{2}-P_{2}^{2}-P_{3}^{2}-\left(q^{-1} t_{0}+q t_{0}^{-1}\right) P_{1} P_{2} P_{3},
\end{aligned}
$$

where

$$
\begin{array}{ll}
\alpha=\left(q^{-1} t_{0}+q t_{0}^{-1}\right) P_{1}+P_{2} P_{3}, & \beta=\left(q^{-1} t_{0}+q t_{0}^{-1}\right) P_{3}+P_{1} P_{2}, \\
\gamma=\left(q^{-1} t_{0}+q t_{0}^{-1}\right) P_{2}+P_{3} P_{1}, & t_{0}^{-1}=P_{0}-t_{0} .
\end{array}
$$

Proof. Write [10, Definition 6.1] and [10, Corollary 6.3] in terms of $A, B, t_{0}$ and $\left\{P_{i}\right\}_{i \in \mathbb{I}}$, using Lemma 16.9 together with (16.1), (16.2).

In this paper we presented several subalgebras of $\hat{H}_{q}$ and $\tilde{\mathfrak{H}}$ by generators and relations. We now compare these presentations. Theorems 4.1 and 4.5 together give a presentation of the subalgebra $\langle A, B, C\rangle$ of $\hat{H}_{q}$ by generators and relations, using the generators $A, B, C$. Theorem 14.1 gives a presentation of the subalgebra $\langle A, B, C, \mathbb{T}\rangle$ of $\hat{H}_{q}$ by generators and relations, using the generators $A, B, C, t_{0}^{ \pm 1}, T_{1}, T_{2}, T_{3}$. Proposition 16.4 gives a presentation of the subalgebra $\mathcal{A}$ of $\tilde{\mathfrak{H}}$ by generators and relations, using the generators $A, B, C, t_{0}$. Proposition 16.5 and Theorem 16.10 each give a presentation of $\mathcal{A}$ by generators and relations, using the generators $A, B, t_{0}$. We now discuss the logical implications between Proposition 16.4, Proposition 16.5, and Theorem 16.10. Proposition 16.5 is discovered from Proposition 16.4 by partially eliminating $C$. Proposition 16.4 is discovered from Proposition 16.5 and the knowledge that $\mathcal{C}=C$. Theorem 16.10 is discovered from Proposition 16.5 by eliminating $\mathcal{C}$. Proposition 16.5 is readily verified using Theorem 16.10. However Proposition 16.5 is not readily discovered using Theorem 16.10 alone. Proposition 16.5 is discovered using Theorem 16.10 and the knowledge that $\mathcal{C}$ simplifies things. Neither $C$ nor $\mathcal{C}$ appears in $[10,11]$.

In [11] Koornwinder discusses an algebra $S(\tilde{\mathfrak{H}})$ known as the spherical subalgebra of $\tilde{\mathfrak{H}}$. In [11, Theorem 3.2] Koornwinder displays an $\mathbb{F}$-algebra isomorphism $\mathrm{AW}\left(3, Q_{0}\right) \rightarrow S(\tilde{\mathfrak{H}})$, where $\operatorname{AW}\left(3, Q_{0}\right)$ is the homomorphic image of $\widetilde{A W}\left(3, Q_{0}\right)$ described in [10, Section 2]. By [11, Section 3] the multiplicative identity of $S(\tilde{\mathfrak{H}})$ is a certain idempotent $P_{\text {sym }}$ in $\tilde{\mathfrak{H}}$. But $P_{\text {sym }} \neq 1$, so $S(\tilde{\mathfrak{H}})$ and $\tilde{\mathfrak{H}}$ do not share the same 1 . Therefore $S(\tilde{\mathfrak{H}})$ is not a subalgebra of $\tilde{\mathfrak{H}}$ according to our convention from Section 1. As far as we know, the results of the present paper are unrelated to $S(\tilde{\mathfrak{H}})$.

\section{Acknowledgements}

The author thanks Kazumasa Nomura for giving this paper a close reading and offering many valuable suggestions.

\section{References}

[1] Alperin R.C., $\mathrm{PSL}_{2}(Z)=Z_{2} \star Z_{3}$, Amer. Math. Monthly 100 (1993), 385-386.

[2] Askey R., Wilson J., Some basic hypergeometric orthogonal polynomials that generalize Jacobi polynomials, Mem. Amer. Math. Soc. 54 (1985), no. 319, iv+55 pages.

[3] Bergman G.M., The diamond lemma for ring theory, Adv. Math. 29 (1978), 178-218. 
[4] Cherednik I., Double affine Hecke algebras, Knizhnik-Zamolodchikov equations, and Macdonald's operators, Int. Math. Res. Not. (1992), 171-180.

[5] Ion B., Sahi S., Triple groups and Cherednik algebras, in Jack, Hall-Littlewood and Macdonald Polynomials, Contemp. Math., Vol. 417, Amer. Math. Soc., Providence, RI, 2006, 183-206, math.QA/0304186.

[6] Ismail M.E.H., Classical and quantum orthogonal polynomials in one variable, Encyclopedia of Mathematics and its Applications, Vol. 98, Cambridge University Press, Cambridge, 2009.

[7] Ito T., Terwilliger P., Double affine Hecke algebras of rank 1 and the $\mathbb{Z}_{3}$-symmetric Askey-Wilson relations, SIGMA 6 (2010), 065, 9 pages, arXiv:1001.2764.

[8] Ito T., Terwilliger P., Weng C.-W., The quantum algebra $U_{q}\left(\mathfrak{s l}_{2}\right)$ and its equitable presentation, J. Algebra 298 (2006), 284-301, math.QA/0507477.

[9] Koekoek R., Lesky P.A., Swarttouw R.F., Hypergeometric orthogonal polynomials and their $q$-analogues, Springer Monographs in Mathematics, Springer-Verlag, Berlin, 2010.

[10] Koornwinder T.H., The relationship between Zhedanov's algebra AW(3) and the double affine Hecke algebra in the rank one case, SIGMA 3 (2007), 063, 15 pages, arXiv:math.QA/0612730.

[11] Koornwinder T.H., Zhedanov's algebra AW(3) and the double affine Hecke algebra in the rank one case. II. The spherical subalgebra, SIGMA 4 (2008), 052, 17 pages, arXiv:0711.2320.

[12] Korovnichenko A., Zhedanov A., Classical Leonard triples, in Elliptic Integrable Systems (2004, Kyoto), Editors M. Noumi, K. Takasaki, Rokko Lectures in Mathematics, no. 18, Kobe University, 2005, 71-84.

[13] Oblomkov A., Double affine Hecke algebras of rank 1 and affine cubic surfaces, Int. Math. Res. Not. 2004 (2004), 877-912, math.RT/0306393.

[14] Sahi S., Nonsymmetric Koornwinder polynomials and duality, Ann. of Math. (2) 150 (1999), 267-282, q-alg/9710032.

[15] Terwilliger P., The universal Askey-Wilson algebra, SIGMA 7 (2011), 069, 24 pages, arXiv:1104.2813.

[16] Terwilliger P., The universal Askey-Wilson algebra and the equitable presentation of $U_{q}\left(\mathfrak{s l}_{2}\right), S I G M A \mathbf{7}$ (2011), 099, 26 pages, arXiv:1107.3544.

[17] Terwilliger P., Vidunas R., Leonard pairs and the Askey-Wilson relations, J. Algebra Appl. 3 (2004), 411426, math.QA/0305356.

[18] Wiegmann P.B., Zabrodin A.V., Algebraization of difference eigenvalue equations related to $U_{q}\left(\mathrm{sl}_{2}\right), N u c l e a r$ Phys. B 451 (1995), 699-724, cond-mat/9501129.

[19] Zhedanov A.S., "Hidden symmetry" of Askey-Wilson polynomials, Theoret. and Math. Phys. 89 (1991), $1146-1157$. 